\title{
Multiscale modelling of diffusion and enzymatic reaction in porous electrodes in Direct Electron Transfer mode
}

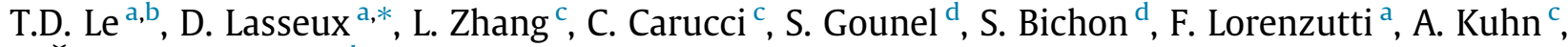 \\ T. Šafarik ${ }^{\mathrm{c}}, \mathrm{N}$. Mano $^{\mathrm{d}}$ \\ a I2M, UMR 5295, CNRS, Univ. Bordeaux, 351, Cours de la Libération, 33405 Talence CEDEX, France \\ ${ }^{\mathrm{b}}$ University of Lorraine, CNRS, LEMTA, F-54000 Nancy, France \\ ${ }^{\mathrm{C}}$ Univ.Bordeaux, CNRS, Bordeaux INP, ISM UMR 5255, Site ENSCBP, 16 Avenue Pey Berland, 33607 Pessac, France \\ ${ }^{\mathrm{d}}$ Centre de Recherche Paul Pascal, CNRS UMR5031, University of Bordeaux, Avenue Albert Schweitzer, 33600 Pessac, France
}

\section{H I G H L I G H T S}

- A macroscopic model for enzymatic porous electrode operating in DET mode is derived.

- The upscaled model is validated with pore-scale direct numerical simulations.

- Macroscopic model predictions are validated by comparisons with experimental data.

\section{A R T I C L E I N F O}

\section{Article history:}

Received 6 June 2021

Received in revised form 20 September

2021

Accepted 27 September 2021

Available online 1 October 2021

\section{Keywords:}

Porous electrode

Direct Electron Transfer

Bilirubin Oxidase

Diffusion reaction

Volume averaging method

\begin{abstract}
A B S T R A C T
This work is dedicated to a multi-scale modelling of coupled diffusion and reaction in a porous microelectrode operating in the Direct Electron Transfer mode. The pore-scale physico-electrochemical unsteady model is developed considering the oxygen reduction, catalyzed by an enzyme coating the pores of the electrode, coupled to the diffusion of oxygen and mass balance of enzymes. This model is formally upscaled to obtain an original closed unsteady macroscopic model operating at the electrode scale, together with the associated closure providing the effective diffusivity tensor. A validation of this model is carried out from a comparison with the solution of the initial 3D pore-scale governing equations considering the bilirubin oxydase as the catalyst. The relevance and accuracy of the macroscale model are proved allowing a considerable simulation speedup. It is further employed to successfully predict experimental voltammetry results obtained with porous gold electrodes functionnalized with a bilirubin oxidase mutant (BOD S362C). This model represents a breakthrough by providing an operational simple way of understanding and further optimizing porous electrodes functioning in DET mode.
\end{abstract}

\section{Introduction}

Over the past two decades, porous electrodes, featuring a high specific surface area (i.e. a large internal solid-fluid interface per unit volume), have been efficiently developed to make miniaturized electro-devices such as bio-batteries, bio-actuators and biosensors (Karajić et al., 2016; Reculusa et al., 2011, 2015; Siepenkoetter et al., 2017). Such electrodes can provide electrical

\footnotetext{
* Corresponding author.

E-mail addresses: didier.lasseux@u-bordeaux.fr (D. Lasseux)
}

current several orders of magnitude larger than simple flat electrodes of the same size (Walcarius and Kuhn, 2008; Szamocki et al., 2006). A classical way to synthesize porous electrodes is to form an ordered assembly of beads, for example by employing the Langmuir-Blodgett technique (Blodgett, 1935) and use it as a template as proposed by Bartlett et al. (Bartlett et al., 2000). Electrodeposition of a conducting material is then performed through the layered bead assembly, followed by beads dissolution, yielding an inverse opale structure constitutive of the electrode (Reculusa and Ravaine, 2003). Due to its versatility, this technique may be employed to obtain porous microstructures with tunable porosity and controllable architecture (Karajić et al., 2016; Reculusa et al., 2015).

For applications in biofuel cells (Leech et al., 2012; de Poulpiquet et al., 2014; Rasmussen et al., 2016; Shleev, 2017; 
Mano and de Poulpiquet, 2018; Solomon et al., 1996; Xiao et al., 2019; Wang et al., 2021), redox reactions can be catalyzed by enzymes which, in order to enhance their lifetime and stability, may be immobilized by entrapping the proteins (Moehlenbrock and Minteer, 2008; Minteer et al., 2012). This can be achieved using different techniques, like entrapment in a conducting polymer matrix occupying a more or less significant fraction of the pores, or adsorption at the pore surfaces (Cadet et al., 2016; Cosnier et al., 2016; Gamella et al., 2018), eventually combined with nano-structuration of the surfaces (de Poulpiquet et al., 2014; Zhao et al., 2017). In the former case, a mediator is employed to shuttle the electrons to and from the electrode's internal surfaces, giving rise to a so-called Mediated Electron Transfer (MET) process. In contrast, in the latter case, when enzymes are adsorbed on the reactive surfaces so that their active centers are positioned within the electron tunnelling distance of the current collector surfaces, the process is referred to as a Direct Electron Transfer (DET). In that case, no mediator is involved. A typical configuration for such a biofuel cell is such that the two electrodes are modified by bioelectrocatalysts. For example at the cathode, the multicopper oxidases (MCOs), such as bilirubin oxidase (BOD), are adsorbed on the electrode surface. They consist of a type 1 copper (T1) site which directly accepts electrons from the electrode, and type 2 and type 3 copper, so-called trinuclear center (TNC site), receiving electrons from T1. The oxygen is then reduced to water at TNC sites (Solomon et al., 1996; Stines-Chaumeil et al., 2017). The process will be further detailed in Section 2.1. In this example, chemical energy is drawn from the oxygen-glucose couple, which naturally exists in physiological fluids for instance, and is converted into electrical energy resulting from oxygen reduction at the cathode and glucose oxidation at the anode.

Modelling of transport and reactions in porous electrodes has often been treated with empirical macroscopic models even in the case without enzymes (see for instance (Levie, 1967; Barcia et al., 2002; Barnes et al., 2014; Ban et al., 2016; Chan et al., 2017)). As a consequence, all the features of the physicoelectrochemical mechanisms (coupling diffusion and reaction in the unsteady regime for example) and of the geometrical structure, in particular for porous electrodes, are not elucidated. More formal approaches were proposed (Vidts and White, 1997; Ferguson and Bazant, 2012; Ender, 2015) without however any explicit closure to relate the pore-scale microstructure to the effective macroscale parameters, in particular, the effective diffusivity. In a recent work, a thorough multiscale model for direct oxygen reduction reaction in a porous electrode was developed by upscaling the pore-scale model and formally obtain a closed macroscale description using the volume averaging method (Le et al., 2017). It was employed to determine the optimal macroscopic thickness of a porous electrode (Le et al., 2019; Le and Lasseux, 2020) and it has been extended to the case of two serial reactions for oxygen reduction (Le et al., 2019).

In the case of reactions catalyzed by enzymes in the DET (or MET) mode, modelling is more complex, and this has motivated empirical analytic expressions of the current density to fit experimental data curves (Tsujimura et al., 2004; Mazurenko et al., 2016). Moreover, in these works, steady-state conditions were assumed for which surface concentrations of enzymes are supposed to be constant and oxygen supply is continuous. Some other studies were carried out for a simple shape non-porous electrode, assuming steady-state (Galceran et al., 2001) or non steady-state conditions (Bartlett and Pratt, 1995). In an interesting work by Do et al. (Do et al., 2014), a non-steady macroscopic model was proposed. The reported model is $1 \mathrm{D}$ and electrode scale equations are not explicitly derived, remaining unclosed since the effective diffusion coefficient is empirically correlated to the porosity of the material. The model was successfully tested to predict experi- mental observations in steady-state conditions. For practical use, a general 3D unsteady and closed macroscopic model operating at the electrode scale is highly desirable, including all the available information of the physico-electrochemical processes at the underlying pore-scale.

In this context, the present work aims at a detailed multiscale modelling of a porous electrode operating in the DET mode, following an upscaling approach. The development represents a complex task due to the coupling of reactions, unsteadiness and non linearity of the overall problem. The purpose is first to provide the set of balance equations, initial and boundary conditions governing the transient coupled process for oxygen diffusion and catalyzed enzymatic reactions in the DET regime within the pores. In this configuration, the pore-scale mass transfer of oxygen is essentially diffusive, while a cathodic oxygen reduction reaction with enzymatic catalysis is considered partially using the framework proposed by Hexter and co-workers (Hexter et al., 2012; Hexter et al., 2014) for the reaction scheme. Upscaling is performed with the volume averaging method (Whitaker, 1999) to obtain a new and original macroscopic model at the electrode scale that is further validated both numerically and with experimental data. This model is fully unsteady, yielding the evolution of both the enzyme and oxygen concentrations. It is formally derived from the porescale equations with a closure providing the effective parameters. This approach has not been reported in the literature so far although this is of prime importance as was raised as a conclusion in a recent review by Rajendran et al. (Rajendran et al., 2017).

The remainder of the article is organized as follows. In Section 2, the pore-scale diffusion/reaction model at the microscale is developed for the enzymatic Direct Electron Transfer regime. In this context, an electrochemical model for the reduction reaction of oxygen catalyzed by BOD is proposed. It is coupled to mass transfer by diffusion of oxygen and mass balance of enzyme under non steadystate conditions. To derive a macroscopic model that is much more effective in terms of computational resources, an upscaling procedure of the pore-scale model is applied, the main steps of which are summarized in Section 3 for the sake of conciseness. More details for this derivation are reported in Appendix A. In Section 4, 3D direct numerical simulations of the pore-scale model are carried out and their results are compared to those obtained from simulations of the 1D macroscopic model to validate the upscaling approach. Experimental details, describing the materials and methods for the electrocatalytic characterization of macroporous gold electrodes are provided in Section 5. The ability of the macroscopic model to predict experimental voltammetry results, obtained from these electrodes modified by BOD adsorption and immersed in a buffer solution, is presented in this section. Conclusions are proposed in Section 6.

\section{Pore-scale diffusion/reaction coupled model}

In this section, the unsteady pore-scale model for diffusion and reaction in a porous electrode, whose internal surface has been modified by the adsorption of BOD enzyme, is developed. The oxygen reduction reaction scheme within the layer containing the enzyme at the electrode surface is assumed to take place in the DET mode. Without any restriction in the physical description of the electrochemical process, the analysis is carried out at the cathode.

The porous electrode, immersed in a reactive solution, occupies the spatial domain $\Omega$ composed of the solid phase $\Omega_{s}$ and the fluid phase $\Omega_{f}$ with the interface denoted by $\Gamma_{s f}=\Omega_{f} \cap \Omega_{s}$ nearby which the oxygen reduction reaction takes place. The electrode is supplied by diffusion of oxygen from the bulk fluid, occupying the domain $\Omega_{e}$, surrounding the electrode. Electron exchange during 
the reduction of oxygen results from a double electron transfer which may be described by a pair of reactions as proposed in Section 2.1.

\subsection{Electrochemical process}

The BOD enzyme is supposed to be immobilized at the surface $\Gamma_{s f}$ of the cathodic electrode to catalyze the oxygen reduction. It contains type 1 copper, forming T1 sites, while TNC sites are composed of type 2 and type 3 copper (Mano and de Poulpiquet, 2018). In the DET mode, electrons from the electrode's internal surface are accepted by type 1 copper (T1 sites) where the enzyme reduction occurs. They are transferred from T1 to the TNC (T2 and T3) sites which remain at a tunneling distance from the pore surfaces and where reduction of oxygen takes place (Tsujimura et al., 2004; Stines-Chaumeil et al., 2017) (see Fig. 1). The reaction scheme at an electrode surface modified with BOD may hence be described following the approach proposed by Hexter and co-workers (Hexter et al., 2012; Hexter et al., 2014) for the reaction of hydrogenases during the reduction of $\mathrm{H}^{+}$to $\mathrm{H}_{2}$. In these works, the electrode is supposed to be continuously supplied with $\mathrm{H}^{+}$so that the mass transfer problem is neglected yielding a description under steady-state conditions. On the contrary, a fully unsteady description is considered here. In the case of oxygen reduction of interest, the catalytic reaction in the DET mode may be written in the following form

$O x+n_{1} e^{-\underset{k_{1 a}}{\stackrel{k_{1 c}}{\rightleftharpoons}}} \operatorname{Re}$ atT1site

$\frac{4}{n_{1}} \mathrm{Re}+\mathrm{O}_{2}+4 \mathrm{H}^{+} \underset{k_{2 a}}{\stackrel{k_{2 c}}{\rightleftharpoons}} \frac{4}{n_{1}} \mathrm{O}+2 \mathrm{H}_{2} \mathrm{O}$ atTNCsite

where $n_{1}$ is the number of transferred electrons involved in the reduction of the enzyme which oxidized and reduced forms are denoted by $O x$ and Re respectively. In the case of BOD, which is further considered for validation and comparison with experiments in Sections 4 and $5, n_{1}=1$ (Tsujimura et al., 2004). Note that the direct reaction pathway for oxygen reduction has been assumed in (1b) as this was confirmed to be the preferential one in experimental observations with the BOD enzyme (Brocato et al., 2012). The electron transfer rate constants $k_{1 a}$ and $k_{1 c}$ are given by the Butler-Volmer relationships

$k_{1 c}=k_{0} \exp \left[-\frac{n_{1} \alpha_{1} F\left(E-E_{O x / R e}^{0}\right)}{R T}\right]$

$k_{1 a}=k_{0} \exp \left[\frac{n_{1}\left(1-\alpha_{1}\right) F\left(E-E_{O x / R e}^{0}\right)}{R T}\right]$

where $k_{0}$ and $\alpha_{1}$ are the standard rate constant and electron transfer coefficient, $E$ and $E_{O x / R e}^{0}$ the electrode potential and the potential of the redox center, $F, R$ and $T$ the Faraday's constant, gas constant and temperature respectively. Similarly, for the oxygen reduction, the catalytic rate constants $k_{2 c}$ and $k_{2 a}$, representative of the internal driving energy within the enzyme, take the following expressions

$k_{2 c}=k / 2 \exp \left[\frac{-n_{2} \alpha_{2} F\left(E_{O x / R e}^{0}-E_{O_{2} / H_{2} O}\right)}{R T}\right]$

$k_{2 a}=k_{2} \exp \left[\frac{n_{2}\left(1-\alpha_{2}\right) F\left(E_{O x / R e}^{0}-E_{\mathrm{O}_{2} / \mathrm{H}_{2} \mathrm{O}}\right)}{R T}\right]$

where $E_{\mathrm{O}_{2} / \mathrm{H}_{2} \mathrm{O}}$ is the thermodynamic equilibrium potential of the couple $\mathrm{O}_{2} / \mathrm{H}_{2} \mathrm{O}, \alpha_{2}$ the electron transfer coefficient associated with this reaction and $n_{2}$ the number of electrons involved. It should be noted that the oxygen reduction in reaction (1b) can be considered as irreversible. For both the validation of the macroscopic model and the comparison with experiments presented in the following sections, the backward reaction in (1b) is ignored and $k_{2 a}$ was hence taken equal to zero for consistency, even if this parameter is formally kept for completeness in the theoretical development reported below, which may straightforwardly be used for an other type of system.

Although four electrons are required for the reduction of $\mathrm{O}_{2}$ in (1b), a different value might be relevant for $n_{2}$ depending on the limiting step governing the electron transfer for this reaction. A further detailed discussion of this feature is beyond the scope of the present work and, as will be seen later, the use of the models derived in the following sections does not explicitly require the value of $n_{2}$ to be specified. In addition, it is worth noticing that the unit of the rate constants $k_{1 c}, k_{1 a}$ and $k_{2 a}$ is $1 / \mathrm{s}$ whereas that of $k_{2 c}$ is $\mathrm{m}^{3} / \mathrm{mol} / \mathrm{s}$. The latter is in contrast with that of the corresponding constant used in the work by Hexter et al. (Hexter et al., 2012) which was restricted to steady conditions that are not retained here as this might be insufficient in practical situations. From the oxygen reduction reaction at TNC sites (see (1b)), the associated reaction rate, $R_{O_{2}}$, can be expressed according to the following Butler-Volmer relationship (Butler, 1932)

$R_{O_{2}}=-k_{2 c} c_{R e} c_{O_{2}}+k_{2 a} c_{O x}$

In the following, a fully coupled diffusion-reaction model is developed, in which concentrations of all species (enzyme and oxygen) are time-dependent to describe the entire unsteady process.

\subsection{Physical model at the pore-scale}

Modelling starts with the initial and boundary value problem governing diffusion of oxygen, which is assumed to be a Fickian process, coupled to oxido-reduction mechanisms inside the pores. Since the enzyme is immobilized at the pore surfaces, no diffusion is taken into account for this species. The pore-scale model can hence be written as follows

$$
\begin{aligned}
& \frac{\partial c_{R e}}{\partial t}=k_{1 c} c_{O x}-k_{1 a} c_{R e}-k_{2 c} c_{R e} c_{O_{2}}+k_{2 a} c_{O x} \quad \text { at } \Gamma_{s f} \\
& c_{O x}+c_{R e}=c_{E}^{t} \quad \text { at } \Gamma_{s f} \\
& \text { I.C.1 } \quad c_{R e}=\mathscr{F}_{R e}(\mathbf{r}) \\
& \frac{\partial c_{\mathrm{O}_{2}}}{\partial t}=\nabla \cdot\left(\mathscr{D}_{\mathrm{O}_{2}} \nabla c_{\mathrm{O}_{2}}\right) \\
& \mathbf{r} \in \Gamma_{s f}, \mathrm{t}=0 \\
& \text { in } \Omega_{f} \\
& \text { B.C.1 }-\mathbf{n} \cdot \mathscr{D}_{\mathrm{O}_{2}} \nabla c_{\mathrm{O}_{2}}=-R_{\mathrm{O}_{2}} \\
& \text { I.C.2 } \quad \mathrm{C}_{\mathrm{O}_{2}}=\mathscr{F}_{\mathrm{O}_{2}}(\mathbf{r}) \\
& \text { B.C.2 } \quad \mathrm{C}_{\mathrm{O}_{2}}=\mathscr{G}_{\mathrm{O}_{2}}(\mathbf{r}, t) \\
& \text { at } \Gamma_{s f} \\
& \mathbf{r} \in \Omega_{f}, \mathrm{t}=0 \\
& \mathbf{r} \in A_{f \mathrm{e}}, \forall \mathrm{t}
\end{aligned}
$$

where $c_{R e}, c_{O x}$ and $c_{O_{2}}$ are the surface concentrations of Re and $O x$ and the bulk oxygen concentration respectively. The total enzyme concentration $c_{E}^{t}$ (see Eq. (5b)) is considered to remain constant at any time whereas mass conservation of $R e$ in Eq. (5a) results from reactions (1). In the oxygen mass conservation Eq. (5d) and in the boundary condition in Eq. (5e), $\mathscr{D}_{\mathrm{O}_{2}}$ is the molecular diffusion coefficient of $\mathrm{O}_{2}$ in the fluid saturating the pores while $\mathbf{n}$ is the unit normal vector to $\Gamma_{s f}$ pointing out of $\Omega_{f}$. The reaction rate, $R_{O_{2}}$, is given by Eq. (4). In the expression of the external boundary condition $B$. C.2, $A_{f \mathrm{e}}=\Omega_{f} \cap \Omega_{e}$ is the entrance and/or exit boundaries of the fluid phase, $\Omega_{f}$, from/into the external bulk fluid, $\Omega_{e}$, in which the electrode is immersed. The solution of Eqs. (5) yields the oxygen and enzymes concentration fields from which the current density, $j$, can be obtained from the reaction rate at T1 sites (1a). It is given by

$j=n_{1} F\left(k_{1 a} c_{R e}-k_{1 c} c_{O x}\right)$ 


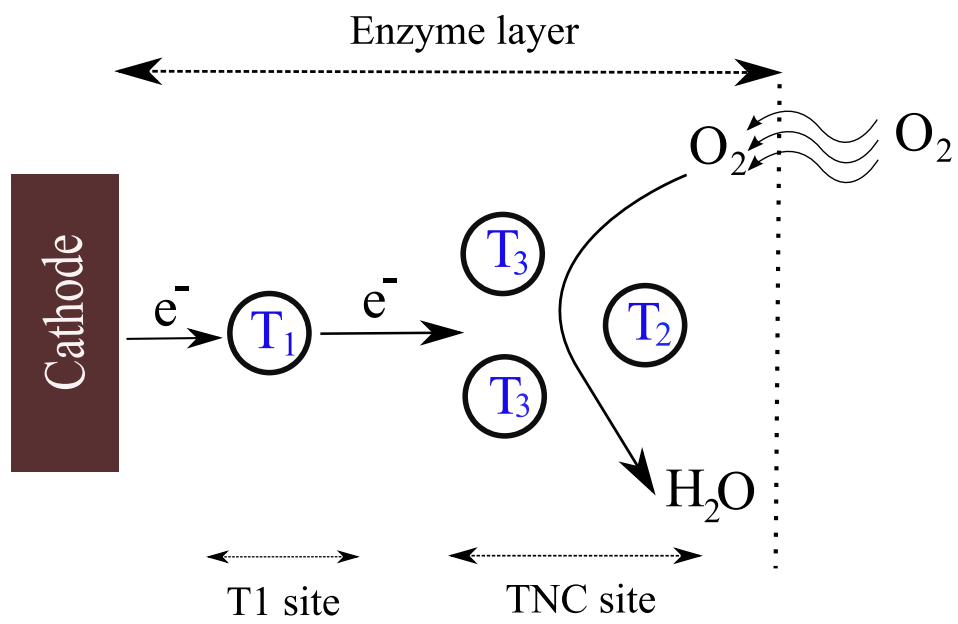

Fig. 1. Schematic representation of electron transfer from the electrode surface to the T1 site and further to a TNC site where oxygen reduction occurs.

that can be further used to compute the total current, $I$, available at the electrode according to

$I=\int_{\Gamma_{s f}} j \mathrm{~d} S$

Although the solution of Eqs. (5) can be sought using a Direct Numerical Simulation (DNS) as will be reported for validation purposes in Section 4.1, a macroscopic model is necessary for the characterization and prediction of the behavior of an electrode. Moreover, as indicated in a previous work dedicated to modelling of electrodes in the absence of enzyme (Le et al., 2017), computational resources required by DNS are substantial and a macroscopic model is much more efficient for practical use. With these objectives in mind, the pore-scale model is upscaled, carrying coupling and non linearities, in order to obtain a macroscopic description that is provided in the next section.

\section{Macroscopic model}

The macroscopic model, operating at the electrode scale, is derived from the initial and boundary value pore-scale problem using the volume averaging method (Whitaker, 1999). For the sake of brevity, only the main result of this procedure is reported below while the details of the formal derivation are provided in Appendix A.

Let $\mathrm{V}$, of measure $V$ and size $r_{0}$, be the averaging volume including the solid and fluid domains $V_{s}$ and $V_{f}$ (of volumes $V_{s}$ and $V_{f}$, respectively) sharing the solid/fluid interface $A_{s f}$ of measure $A_{s f}$. The porosity, $\varepsilon_{f}$, and specific area, $a_{v}$, of the porous material are respectively defined by

$$
\varepsilon_{f}=V_{f} / V(8 \mathrm{a})
$$$$
a_{v}=A_{s f} / V(8 \mathrm{~b})
$$

The upscaling process makes use of the superficial, intrinsic and area averages of the concentration fields for oxygen and reduced enzyme that are respectively defined by

$$
\begin{aligned}
& \left.\left\langle c_{O_{2}}\right\rangle\right|_{\mathbf{x}}=\left.\frac{1}{V} \int_{\mathrm{V}_{f}(\mathbf{x})} c_{O_{2}}\right|_{\mathbf{x}+\mathbf{y}} \mathrm{d} V \\
& \left.\left\langle c_{O_{2}}\right\rangle^{f}\right|_{\mathbf{x}}=\left.\frac{1}{V_{f}} \int_{\mathrm{V}_{f}(\mathbf{x})} c_{O_{2}}\right|_{\mathbf{x}+\mathbf{y}} \mathrm{d} V \\
& \left.\left\langle c_{X}\right\rangle_{s f}\right|_{\mathbf{x}}=\left.\frac{1}{A_{s f}} \int_{\mathrm{A}_{s f}(\mathbf{x})} c_{X}\right|_{\mathbf{x}+\mathbf{y}} \mathrm{d} A, \quad X=O_{2}, R e
\end{aligned}
$$

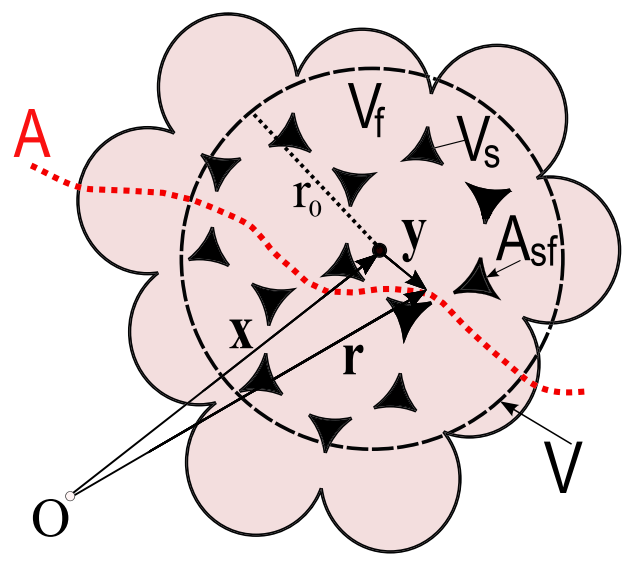

Fig. 2. Averaging volume for a two-phase system.

with the straightforward relationship $\left\langle c_{\mathrm{O}_{2}}\right\rangle=\varepsilon_{f}\left\langle c_{\mathrm{O}_{2}}\right\rangle^{f}$ and where $\mathbf{x}$ locates the centroid of the averaging volume while $\mathbf{y}=\mathbf{r}-\mathbf{x}$ locates any point within $\vee_{f}$ relative to $\mathbf{x}$ (see Fig. 2). For the sake of simplicity, the subscript referring to the location may be dropped, unless necessary. The derivation of the upscaled model is subject to a scale hierarchy defined by $\ell_{p} \ll r_{0} \ll L, \ell_{p}$ being the characteristic pore size and $L$ the macroscopic size of the electrode. The upscaling procedure is carried out according to the four main steps reported in Appendix A and under the constraints on the kinetic number, $\mathrm{Ki}$, and time scale respectively given by

$K i=\frac{k_{2 c} \ell_{p} c_{E}^{t}}{\mathscr{D}_{\mathrm{O}_{2}}} \ll 1$

$t \gg \mathcal{O}\left[\frac{\ell_{p}^{2}}{\mathscr{D}_{O_{2}}}, \frac{1}{k_{1 c}+k_{1 a}+k_{2 a}+k_{2 c}\left\langle c_{O_{2}}\right\rangle^{f}}\right]$

Under these circumstances, the macroscopic model, including the enzyme and oxygen effective mass conservation equations operating at the electrode scale, can be written as

$\frac{\partial\left\langle c_{R e}\right\rangle_{s f}}{\partial t}=-\left(k_{1 c}+k_{1 a}+k_{2 a}\right)\left\langle c_{R e}\right\rangle_{s f}-k_{2 c}\left\langle c_{R e}\right\rangle_{s f}\left\langle c_{O_{2}}\right\rangle^{f}+\left(k_{1 c}+k_{2 a}\right) c_{E}^{t}$

$\left\langle c_{O x}\right\rangle_{s f}=c_{E}^{t}-\left\langle c_{R e}\right\rangle_{s f}$

$\varepsilon_{f} \frac{\partial\left\langle c_{O_{2}}\right\rangle^{f}}{\partial t}=\nabla \cdot\left(\varepsilon_{f} \mathbf{D}_{e f f} \cdot \nabla\left\langle c_{O_{2}}\right\rangle^{f}\right)$

$-k_{2 c} a_{v}\left\langle c_{O_{2}}\right\rangle^{f}\left\langle c_{R e}\right\rangle_{s f}-k_{2 a} a_{v}\left(\left\langle c_{R e}\right\rangle_{s f}-c_{E}^{t}\right)$ 
The constraints expressed in (10) indicate that, strictly speaking, the averaged model can provide accurate predictions of the oxygen and enzyme average concentrations in the mass transfer limited regime (10a) and for times larger than the overall reaction kinetics characteristic time (10b). First, it must be noted that, despite the closure can be treated as a steady problem with these assumptions, the macroscale model remains unsteady, and this is because the time scales of variation of the average and pore-scale concentrations are different. Secondly, it must be emphasized that these constraints are usually overly severe and, most of the time, the range of applicability of the model can be extended beyond these constraints, i.e., for $K i \sim 1$ and $t \sim \mathcal{O}\left[\frac{\ell_{p}^{2}}{\mathscr{D}_{2}}, \frac{1}{k_{1 c}+k_{1 a}+k_{2 a}+k_{2 c}\left\langle c_{O_{2}}\right\rangle}\right]$ (see for instance (Valdés-Parada et al., 2017, 2020) for similar but somewhat different problems). Nevertheless, this deserves a careful analysis that is beyond the scope of this work.

In the macroscopic diffusion-reaction Eq. (11c), $\mathbf{D}_{\text {eff }}$ is the effective diffusion tensor (that is symmetric (Lasseux and ValdésParada, 2017)), given by

$\left.\mathbf{D}_{\text {eff }}=\mathscr{D}_{\mathrm{O}_{2}} \mathbf{I}+\frac{1}{V_{f}} \int_{\mathrm{A}_{s f}} \mathbf{n b} d A\right)$

in which $\mathbf{I}$ is the identity tensor and $\mathbf{n b}$ denotes the outer product between the two vectors $\mathbf{n}$ and $\mathbf{b}$, the latter being solution of the following closure problem (see also Eqs. (A.33) in Appendix A)

$\nabla^{2} \mathbf{b}=0 \quad$ in $\mathrm{V}_{f}$

$\mathbf{n} \cdot \nabla \mathbf{b}=-\mathbf{n} \quad$ at $\mathrm{A}_{s f}$

$\langle\mathbf{b}\rangle^{f}=0$

$\mathbf{b}(\mathbf{r})=\mathbf{b}\left(\mathbf{r}+\ell_{i} \mathbf{e}_{i}\right) \quad i=1,2,3$

This problem is intrinsic to the porous structure, which means that its solution only depends on the microgeometry of the pore space. To obtain this result, the averaging domain, $\mathrm{V}$, was taken as a Representative Elementary Volume of the porous structure that was further considered as pseudo periodic so that the closure problem is solved on a unit cell whose periodic lattice vectors are $\ell_{i} \mathbf{e}_{i}$ $(i=1,2,3)$.

It should be noted that the closure performs the essential link between the micro- and macroscale models. Indeed, the closure problem given in Eqs. (13) contains the nonredundant information (in particular the microsctructure implied in $\mathrm{V}_{f}$ and $\mathrm{A}_{s f}$ ) that is essential in the macroscopic model. As a result, Eqs. (11), together with the initial and boundary conditions on $\left\langle c_{R e}\right\rangle_{s f}$ and $\left\langle c_{O_{2}}\right\rangle^{f}$, form the new macroscopic effective model of coupled diffusion-reaction for a porous electrode operating in the DET mode.

The expression of the current from the macroscopic surface average of the reduced enzyme concentration can now be derived. The surface average of Eq. (6) yields

$\langle j\rangle_{s f}=n_{1} F\left(k_{1 a}\left\langle c_{R e}\right\rangle_{s f}-k_{1 c}\left(c_{E}^{t}-\left\langle c_{R e}\right\rangle_{s f}\right)\right)$

from which the current per unit volume of the unit cell representative of the medium, $i_{v}$, can be deduced as $i_{v}=\langle j\rangle_{s f} A_{s f} / V=a_{v}\langle j\rangle_{s f}$. The total current, $I$, delivered by the electrode is hence given by $I=\int_{\Omega} i_{v} \mathrm{~d} V$, i.e.

$I=a_{v} \int_{\Omega}\langle j\rangle_{s f} \mathrm{~d} V$

\section{Numerical results and discussions}

The relevance of the prediction obtained with the new original macroscopic model is assessed by a comparison with DNS of the pore-scale problem. This is carried out on a model porous structure whose unit cell, of size $\ell_{R}$, is a face-centered cubic (FCC) arrangement of spherical pores, of diameter $d_{s} \equiv \ell_{p}$, interconnected to each other through circular windows of diameter $d_{c}$ (see Fig. 3). The objective of this procedure is to validate the macroscopic model so that it can be used in place of the pore-scale model with an expected important gain in performance.

\subsection{Direct numerical simulation of the pore-scale model}

The 3D numerical voltammetry experiments were performed on a computational domain represented in Fig. 3. The porous electrode is composed of periodic FCC unit cells in the $z$-direction, between $z=-L_{e}$, where the electrically conducting electrode support is positioned, and $z=0$, where the electrode is in contact with the bulk fluid saturating the pores. The fluid region, $\Omega_{e}$, between $z=0$ and $z=L_{N}$, corresponds to the diffusion layer where oxygen molecular diffusion takes place. Periodic boundary conditions are applied in the $x$ and $y$ directions. This is justified by the fact that the electrode length is supposed to be much larger than $\ell_{R}$ and that the same holds for its lateral extension, if the electrode is a plane one. If it is circular, $L_{e}$ is supposed to be much smaller than its mean radius. A zero flux condition is imposed at $z=-L_{e}$ while a constant oxygen concentration, $c_{\mathrm{O}_{2}}=c_{\mathrm{O}_{2}}^{0}$, is considered at the interface of the diffusion layer with the rest of the bulk fluid, $z=L_{N}$. The initial concentration of oxygen is supposed to be uniform equal to $c_{\mathrm{O}_{2}}^{0}$. In addition, only oxidized enzyme is assumed to be present at the initial state, leading to $c_{R e}=0$ at $t=0$. The pore-scale problem in Eqs. (5), coupled to the diffusion of $\mathrm{O}_{2}$ in the diffusion layer, can be rewritten in a dimensionless form using $\ell_{R}, \frac{\ell_{R}^{2}}{\mathscr{D}_{O_{2}}}, c_{\mathrm{O}_{2}}^{0}$ and $c_{E}^{t}$ as the reference length, time, oxygen and enzyme surface concentrations. Denoting dimensionless quantities with the superscript $*$, this coupled non-linear problem is given by

$\frac{\partial c_{O_{2}}^{*}}{\partial t^{*}}=\nabla^{* 2} c_{O_{2}}^{*} \quad$ in $\Omega_{f} \cup \Omega_{e}$
B.C. $1 \quad-\mathbf{n} \cdot \nabla^{*} c_{O_{2}}^{*}=0 \quad$ at $z$

B.C. $1-\mathbf{n} \cdot \nabla^{*} c_{O_{2}}^{*}=0 \quad$ at $z^{*}=-L_{e}^{*}$

B.C.2 $c_{\mathrm{O}_{2}}^{*}=1 \quad$ at $z^{*}=L_{N}^{*}$

B.C.3 $-\mathbf{n} \cdot \nabla^{*} c_{O_{2}}^{*}=\frac{\ell_{R} c_{E}^{t}}{\mathscr{D}_{O_{2}}}\left(k_{2 c} c_{R e}^{*} c_{O_{2}}^{*}-\frac{k_{2 a}}{c_{O_{2}}^{0}}\left(1-c_{R e}^{*}\right)\right)$ at $\Gamma_{s f}$

$\frac{\partial c_{R e}^{*}}{\partial t^{*}}=\frac{\ell_{R}^{2}}{\mathscr{D}_{O_{2}}}\left(-\left(k_{1 c}+k_{1 a}+k_{2 a}+k_{2 c} c_{O_{2}}^{0} c_{O_{2}}^{*}\right) c_{R e}^{*}+k_{1 c}+k_{2 a}\right)$ at $\Gamma_{s f}$

I.C. $1 c_{O_{2}}^{*}=1 \quad$ in $\Omega_{f} \cup \Omega_{e}$ at $t^{*}=0$

I.C. $2 c_{R e}^{*}=0$ at $\Gamma_{s f}$ at $t^{*}=0$

$c_{O_{2}}^{*}\left(x^{*}, y^{*}, z^{*}\right)=c_{O_{2}}^{*}\left(x^{*}+1, y^{*}+1, z^{*}\right), \quad$ in $\Omega_{f}$

$\mathbf{n} \cdot \nabla^{*} c_{O_{2}}^{*}\left(x^{*}, y^{*}, z^{*}\right)=\mathbf{n} \cdot \nabla^{*} c_{O_{2}}^{*}\left(x^{*}+1, y^{*}+1, z^{*}\right), \quad$ in $\Omega_{f}$

$c_{R e}^{*}\left(x^{*}, y^{*}, z^{*}\right)=c_{R e}^{*}\left(x^{*}+1, y^{*}+1, z^{*}\right), \quad$ at $\Gamma_{s f}$

Voltammetry simulation, with a scan-rate $r_{E}=5 \mathrm{mV} / \mathrm{s}$ and an initial potential value of $0.6 \mathrm{~V}$, was carried out with $L_{e}^{*}=10$ and $L_{N}=20 \mu m$ (i.e. $L_{N}^{*} \simeq 12$ ). Values of the other physical parameters are reported in Table 1 . It should be noted that $k_{2 a}$ is taken equal to zero since the reduction of $\mathrm{O}_{2}$ is considered as irreversible. Since BOD is the enzyme for the catalytic reaction, $n_{1}=1$ (Tsujimura et al., 2004).

The software COMSOL Multiphysics (ver. 5.4), with physicscontrolled mesh including extremely fine grid blocks composed of $10^{7}$ tetrahedral elements in the overall domain, was used to solve this problem. The typical computational time is about $12 \mathrm{~h}$ on a Dell PowerEdge 430-2 processors Intel Xeon E5-2630v3.

Dimensionless oxygen concentration fields obtained from DNS in $\Omega_{f}$ and $\Omega_{e}$ are represented in Fig. 4 at $t=10 \mathrm{~s}, 55 \mathrm{~s}$ and $70 \mathrm{~s}$, highlighting the coupled diffusion-reaction process inside the por- 


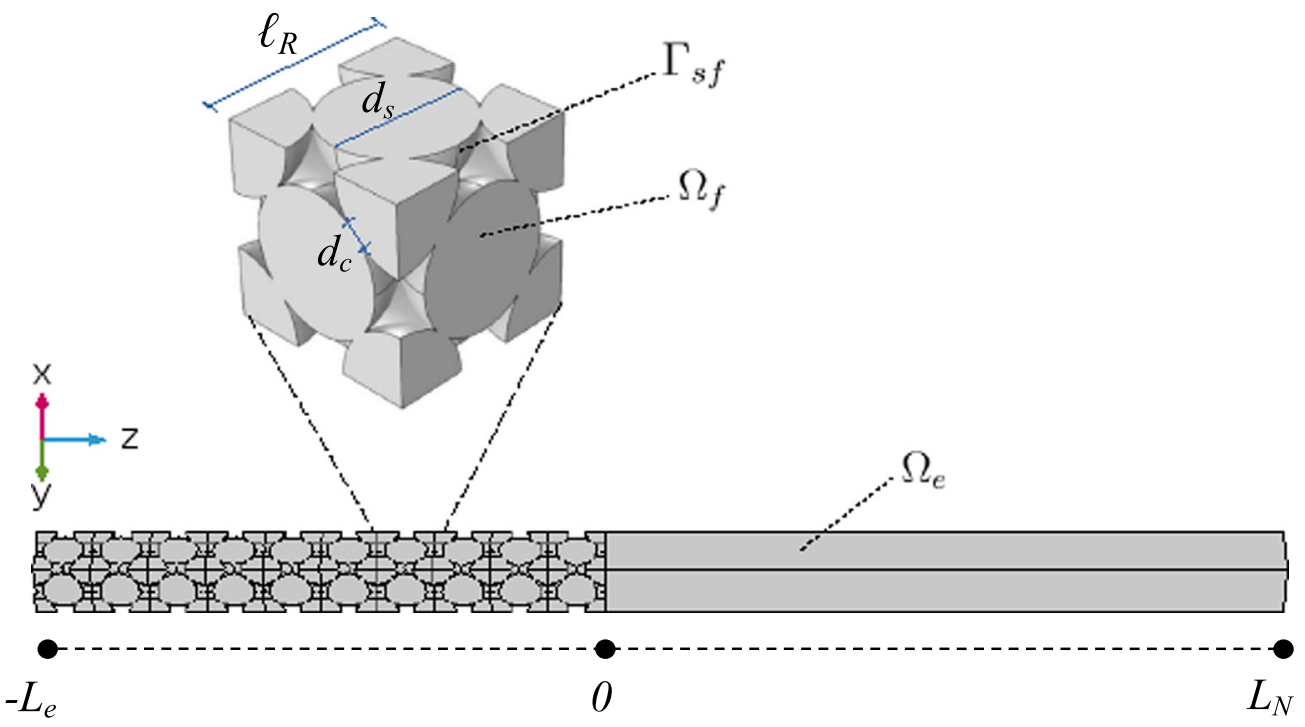

Fig. 3. 3D domain for the direct numerical simulation.

Table 1

Parameters used for the simulations.

\begin{tabular}{cccc}
\hline Parameter & Symbol & Value & Unit \\
\hline Ideal gas constant & $R$ & 8.314 & $\mathrm{Jmol}^{-1} \mathrm{~K}^{-1}$ \\
Faraday's constant & $F$ & 96485.33 & $\mathrm{Cmol}^{-1}$ \\
Number of transferred electron & $n_{1}$ & 1 & - \\
Electron transfer coefficient & $\alpha_{1}$ & 0.5 & - \\
Standard potential vs. $E_{\mathrm{Ag} / \mathrm{AgCl}}^{0}$ & $E_{\mathrm{Ox} / \mathrm{Re}}^{0}$ & 0.41 & $\mathrm{~V}$ \\
Equilibrium potential vs. $E_{\mathrm{Ag} / \mathrm{AgCl}}^{0}$ & $E_{\mathrm{O}_{2} / \mathrm{H}_{2} \mathrm{O}}$ & 0.619 & $\mathrm{~V}$ \\
Scan rate & $r_{E}$ & 5 & $\mathrm{mVs}^{-1}$ \\
Temperature & $T$ & 298 & $\mathrm{~K}$ \\
Bulk concentration & $c_{\mathrm{O}_{2}}^{0}$ & 1.2 & $\mathrm{~mol} \mathrm{~m}^{-3}$ \\
Diffusion coefficient & $c_{E}^{t}$ & 10 & $\mathrm{~mol} \mathrm{~m}^{-2}$ \\
Standard rate constant & $\mathscr{D}_{\mathrm{O}_{2}}$ & $2 \times 10^{-9}$ & $\mathrm{~m}^{2} \mathrm{~s}^{-1}$ \\
Electron transfer rate constant & $k_{0}$ & 10 & $\mathrm{~s}^{-1}$ \\
Spherical pore diameter & $k_{2 c}$ & 485.83 & $\mathrm{~m}^{3} \mathrm{~mol}^{-1} \mathrm{~s}^{-1}$ \\
Relative pore connection window size & $d_{s} / d_{s}$ & 1.17 & $\mu \mathrm{m}$ \\
Size of the periodic unit cell & $\ell_{R}$ & 1.678 & $\mu \mathrm{m}$
\end{tabular}

ous electrode and showing the oxygen concentration decrease with time. In this configuration, $K i=10^{-3}$, a value that satisfies the constraint in (10a). These results can now be directly compared to simulations of the macroscopic model and this is the object of the following section.

\subsection{Solution of the macroscopic model and comparisons with DNS}

Our aim now is to explore the relevance of the macroscopic model, its performance to predict the average concentrations and the current delivered by the electrode. Since the FCC structure under consideration here is isotropic, $\mathbf{D}_{\text {eff }}=\mathscr{D}_{\text {eff }} \mathbf{I} \mathbf{I} \mathbf{I}$ being the identity tensor) and the macroscopic model reduces to $1 \mathrm{D}$. The macroscopic computational domain constituted by the 1D effective medium for the electrode and the diffusion layer is represented in Fig. 5.

Using the same reference quantities, the dimensionless macroscopic problem can be written as

$$
\begin{aligned}
& l \varepsilon_{f} \frac{\partial\left\langle c_{O_{2}}^{*}\right\rangle^{f}}{\partial t^{*}}=\varepsilon_{f} \mathrm{D}_{e f f}^{*} \frac{\partial^{2}\left\langle c_{O_{2}}^{*}\right\rangle^{f}}{\partial z^{* 2}} \\
& -\frac{a_{v} \ell_{R}^{2} c_{E}^{t}}{\mathscr{D}_{O_{2}} c_{O_{2}}^{0}}\left(k_{2 c} c_{O_{2}}^{0}\left\langle c_{O_{2}}^{*}\right\rangle^{f}\left\langle c_{R e}^{*}\right\rangle_{s f}+k_{2 a}\left(\left\langle c_{R e}^{*}\right\rangle_{s f}-1\right)\right) \text { in } \Omega \\
& \frac{\partial\left\langle c_{R e}^{*}\right\rangle_{s f}}{\partial t^{*}}=\frac{\ell_{R}^{2}}{\mathscr{D}_{O_{2}}}\left(-\left(k_{1 c}+k_{1 a}+k_{2 a}\right)\left\langle c_{R e}^{*}\right\rangle_{s f}\right. \\
& \left.-k_{2 c} c_{O_{2}}^{0}\left\langle c_{O_{2}}^{*}\right\rangle^{f}\left\langle c_{R e}^{*}\right\rangle_{s f}+k_{1 c}+k_{2 a}\right) \text { in } \Omega
\end{aligned}
$$

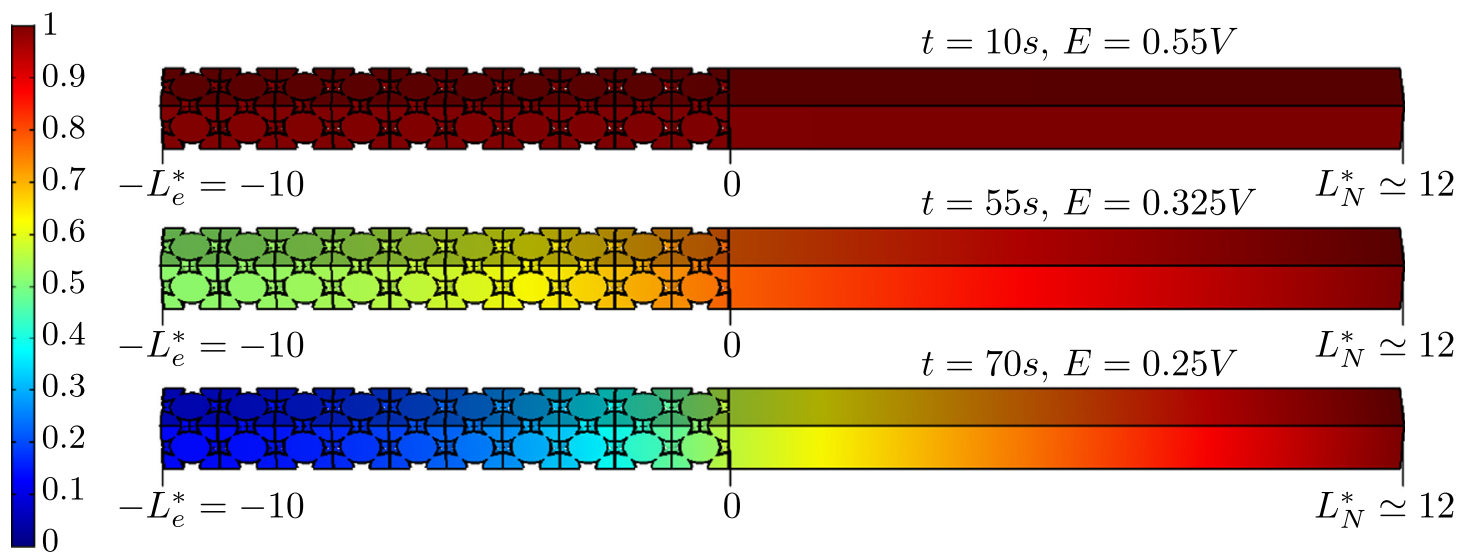

Fig. 4. Normalized $\mathrm{O}_{2}$ concentration fields, $c_{\mathrm{O}_{2}}^{*}$, at $t=10 \mathrm{~s}, 55 \mathrm{~s}$ and $70 \mathrm{~s}$, the corresponding potential values being $E=0.55 V, 0.325 V$ and $E=0.25 V$, respectively. 


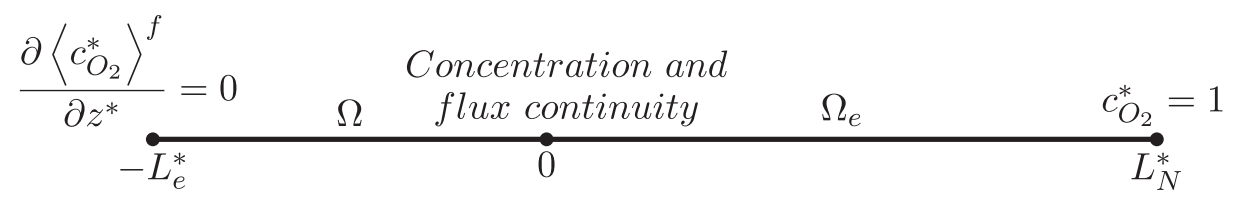

Fig. 5. 1D configuration for the macroscopic numerical simulation.

$$
\begin{aligned}
& \text { B.C. } 1\left\langle c_{O_{2}}^{*}\right\rangle^{f}=c_{O_{2}}^{*} \quad \text { at } z^{*}=0 \\
& \text { B.C.2 } \varepsilon_{f} \mathrm{D}_{e f f}^{*} \frac{\partial\left\langle c_{\mathrm{O}_{2}}^{*}\right\rangle^{f}}{\partial z^{*}}=\frac{\partial c_{\mathrm{O}_{2}}^{*}}{\partial z^{*}} \quad \text { at } z^{*}=0 \\
& \frac{\partial c_{\mathrm{O}_{2}}^{*}}{\partial t^{*}}=\frac{\partial^{2} c_{\mathrm{O}_{2}}^{*}}{\partial z^{* 2}} \quad \text { in } \Omega_{e} \\
& \begin{array}{ll}
\text { B.C.3 } & \frac{\partial\left\langle c_{O_{2}}^{*}\right\rangle^{f}}{\partial z^{*}}=0 \quad \text { at } z^{*}=-L_{e}^{*} \\
\text { B.C.4 } & c_{O_{2}}^{*}=1
\end{array} \\
& \text { I.C. } 1\left\langle c_{\mathrm{O}_{2}}^{*}\right\rangle^{f}=1 \quad \text { in } \Omega \text { at } t^{*}=0 \\
& \text { I.C. } 2\left\langle c_{R e}^{*}\right\rangle_{s f}=0 \quad \text { in } \Omega \text { at } t^{*}=0 \\
& \text { I.C.3 } c_{O_{2}}^{*}=1 \quad \text { in } \Omega_{e} \text { at } t^{*}=0
\end{aligned}
$$

In Eq. (17a), $\mathrm{D}_{\text {eff }}^{*}$ represents the dimensionless effective diffusion coefficient, $\mathrm{D}_{\text {eff }}^{*}=\mathrm{D}_{\text {eff }} / \mathscr{D}_{\mathrm{O}_{2}}$. Prior to the solution of the above model, the closure problem in Eqs. (13) was solved over a periodic FCC unit cell. Both the closure and macroscopic problems were solved with COMSOL Multiphysics (ver. 5.4).

Voltammetry simulation using the 1D-macroscopic effective model in Eqs (17) was carried out in the same conditions as for the 3D DNS at the microscale presented in Section 4.1, i.e. using the parameters indicated in Table 1 with $L_{e}^{*}=10$ and $L_{N}^{*} \simeq 12$. For this microstructure, $\varepsilon_{f}=0.763, a_{v}^{*}=5.985$ and $\varepsilon_{f} \mathrm{D}_{\text {eff }}^{*}=0.364$. The mesh used for this simulation is made of $\sim 800$ elements. The pore-scale fields of $c_{\mathrm{O}_{2}}^{*}$ and $c_{\mathrm{Re}}^{*}$ were averaged in each unit cell over $\mathrm{V}_{f}$ and $\mathrm{A}_{s f}$ respectively and then compared to $\left\langle c_{O_{2}}^{*}\right\rangle^{f}$ and $\left\langle c_{R e}^{*}\right\rangle_{s f}$ obtained from the solution of the macroscopic model.

The dimensionless oxygen concentration profiles inside the porous electrode, obtained from the 3D-DNS and 1D simulation, are represented at the three times $t=10 s, 55 s$ and $70 s$ in Fig. 6. This concentration decreases with time as a result of the reduction reaction while its gradient increases at the electrode-diffusion layer interface $\left(z^{*}=0\right)$ and hence inside the electrode. As shown on this figure, the agreement between the two approaches is excellent. Similarly, the dimensionless reduced enzyme surface concentration profiles obtained with both the micro and macro-scale models are reported in Fig. 7. Again, the comparison between the two shows an excellent agreement, confirming the validity of the new macroscopic model derived in this work. As a final important check, the interest must now be focused on the current curves in voltamograms. The current was computed by making use of Eqs. (6) and (7) in the microscale approach and with Eqs. (14) and (15) involving the average reduced enzyme concentration obtained from the solution of the macroscopic model. In Fig. 8, the current per unit cross-sectional area of the electrode is represented versus the scanning potential ranging from $0.6 \mathrm{~V}$ to $0.1 \mathrm{~V}$. A special attention must be dedicated to the mesh refinement for the 3D DNS in COMSOL Multiphysics. A tetrahedral mesh made of $\sim 3.3 \times 10^{5}$ elements for the domain under consideration in Fig. 3 was used to obtain converged results. As can be seen in Fig. 8, the agreement between the two approaches is again excellent. This confirms the perfect agreement between the two approaches and completes the validation of the new macroscopic model which provides a powerful tool to predict the macroscopic behavior of a porous electrode operating in the DET mode. In addition it must be emphasized that this original macroscopic approach allows a considerable computational speedup. Indeed, in the case under consideration in this section, the macroscale solution requires only $4 \mathrm{~s}$, which, compared to the corresponding 3D DNS with the above mentioned mesh ( $85 \mathrm{~min}$ ), represents a speedup of about 1300 .

In the following section, the ability of the macroscopic model to predict the current-to-potential relationship characteristics of an electrode is reported through comparisons with experimental voltammetry results obtained with a porous gold electrode coated with BOD as the bioelectrocatalyst at the cathode.

\section{Comparison with experimental data}

In this section, the capability of the macroscopic model to predict voltammetry experimental data is investigated. To this purpose, experiments were carried out on porous gold electrodes.

\subsection{Experimental details}

The three main steps of the experimental protocol consist in the electrode synthesis, the enzyme immobilization and the electrocatalytic characterization.

\section{Electrode Synthesis}

Cylindrical macroporous gold electrodes were prepared using a Langmuir-Blodgett technique as shortly described in the introduction and detailed in (Bartlett et al., 2000; Reculusa et al., 2015; Reculusa and Ravaine, 2003). In brief, silica beads with a diameter of $1170 \mathrm{~nm}\left(d_{s}=1.2 \mu \mathrm{m}\right.$ is taken as an accurate enough value) were spread from an ethanol/chloroform $(20 \% \mathrm{v} / \mathrm{v}-80 \% \mathrm{v} / \mathrm{v})$ suspension at the water-air interface of a Langmuir-Blodgett trough. An optimal monolayer of silica beads was obtained at the waterair interface after compression by closing the moveable barriers. Then, multiple silica particle layers were transferred onto gold wires of $250 \mu \mathrm{m}$ in diameter and $4 \mathrm{~cm}$ in length. This was achieved by repeating the dipping and withdrawing process at a speed of 1.2 $\mathrm{mm} / \mathrm{min}$. After the formation of the silica template layers on the surface, Elevate ${ }^{\circledR}$ Gold 7990 was used for the gold electrodeposition over a length of $2 \mathrm{~cm}$ by applying a constant potential of $-0.6 \mathrm{~V}$. The number of macroporous layers is controlled by following the current oscillations. After dissolving the silica particles in $5 \%$ hydrofluoric acid for several minutes, the final macroporous gold electrode is obtained as an inverse opale gold structure. Four electrodes were prepared, having 3, 7, 11 and 19 half-layers (HL) of pores. At this stage, the internal pore surfaces were coated with a genetically engineered variant of Bilirubin oxidase from $M$. oryzae whose serine in position 362 was replaced by a cysteine (BOD S362C) (Al-Lolage et al., 2019). This was peformed over the porous zone being $2 \mathrm{~cm}$ long, corresponding to a surface area of the initial wire of $15 \mathrm{~mm}^{2}$. The actual active surface area depends on the thickness of the porous layers of the electrode. There is a linear correspondence between the active area of the porous electrode and the number of porous half layers, as reported recently (Zhang et al., 2019).

\section{Enzyme Immobilization}

A $0.1 \mathrm{mM}$ BOD S362C $(516 \pm 38 \mathrm{U} / \mathrm{mg})$ solution was prepared in $50 \mathrm{mM}$ phosphate buffer ( $\mathrm{pH}$ 6.0). Since the cysteine residue on the outer surface of bilirubin oxidase is close to the T1 active center, the interaction between the thiol group on the cysteine residue and the gold surface allows the formation of a self-assembled 


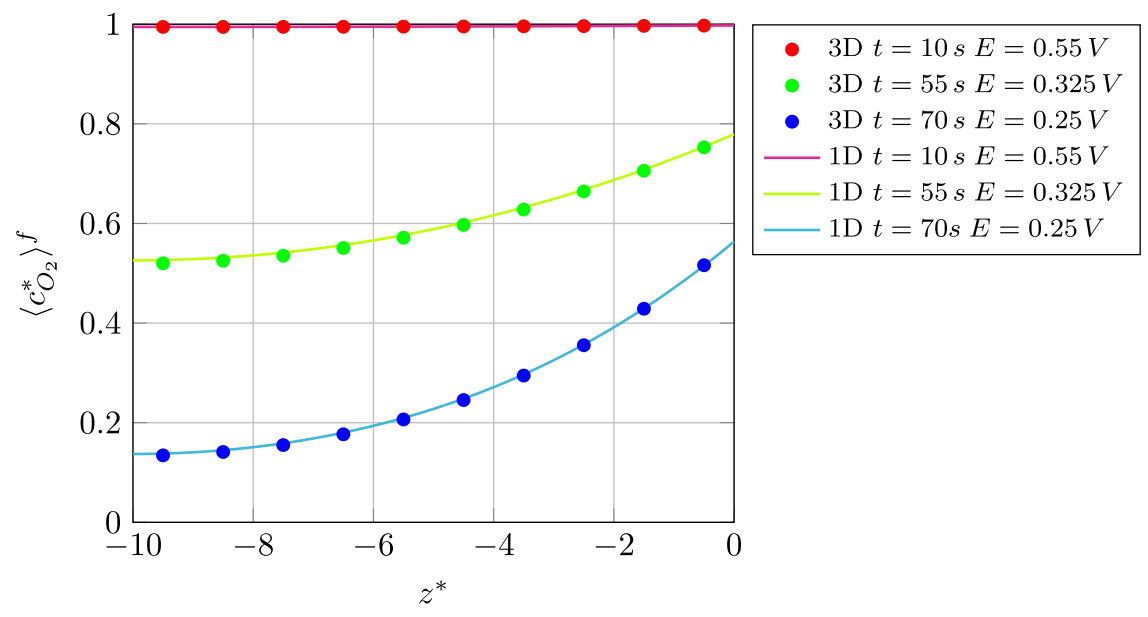

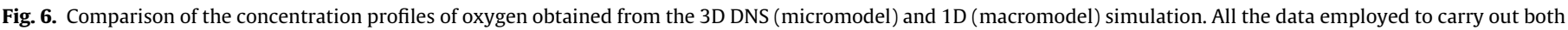
the pore-scale DNS and the macroscale simulation are mentioned in the text above and in Table 1.

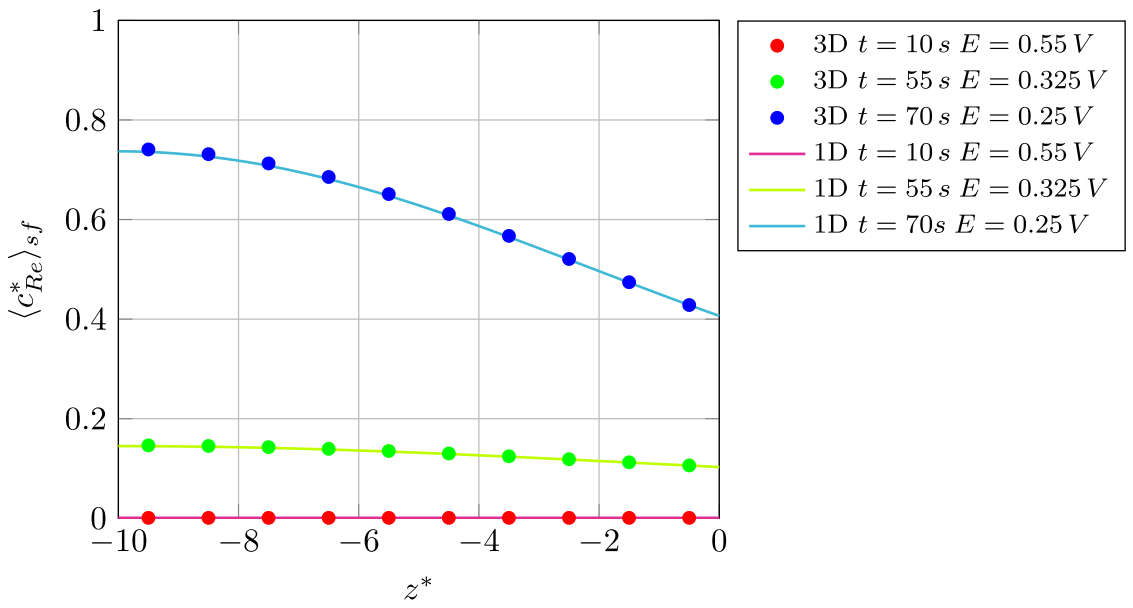

Fig. 7. Comparison of reduced enzyme concentration profiles obtained from the 3D DNS (micromodel) and 1D (macromodel) simulations.

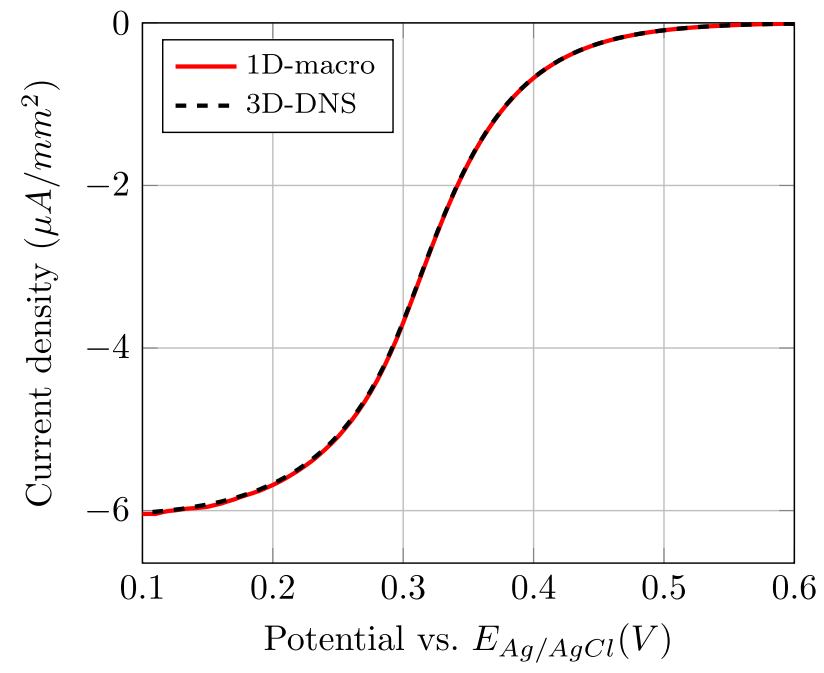

Fig. 8. Comparison between the current per unit electrode cross-sectional area versus the scanning potential during voltammetry obtained from the 3D DNS and 1D macroscopic model. monolayer of BOD S362Cs on the internal surface of the pores. This allows a proper orientation of the BOD on the electrode surface and favors the interfacial electron transfer. The macroporous gold electrode was dipped into the enzyme solution after being treated with plasma for $15 \mathrm{~min}$. Vacuum was then applied for $3 \mathrm{~min}$ to ensure an efficient penetration of enzymes into the whole porous structure. The electrode was kept at $4{ }^{\circ} \mathrm{C}$ overnight. After rinsing with distilled water and with $0.1 \mathrm{M}$ PBS pH 7.2 under stirring, the electroenzymatic $\mathrm{O}_{2}$ reduction was performed with the BOD modified electrodes.

\section{Electrocatalytic characterization}

All electrochemical measurements were performed with an Autolab PGSTAT101 potentiostat monitored by a PC running Nova 1.6. A $\mathrm{Ag} / \mathrm{AgCl}$ (3 M KCl) was used as a reference electrode, and a cylindrical carbon sheet was employed as an auxiliary electrode. For the electrochemical reduction, the buffer solution was purged with $\mathrm{O}_{2}$ for $15 \mathrm{~min}$. Then, the electrodes were dipped into the buffer solution maintained at ambient temperature while a slow flow of $\mathrm{O}_{2}$ was kept above it inside a sealed cell for the $\mathrm{O}_{2}$ concentration to remain constant in the buffer. Voltammetry experiments were carried out in the potential range from $0.6 \mathrm{~V}$ to $0.15 \mathrm{~V}$ with a scan rate of $5 \mathrm{mV} / \mathrm{s}$. The temperature was $25^{\circ} \mathrm{C}$ for these experiments. Each experiments was at least performed in triplicate. 
Table 2

Parameters fitted on the voltammetry results obtained with the $11 \mathrm{HL}$ electrode and used for all the other predictions employing the macroscopic model.

\begin{tabular}{cccc}
\hline$k_{0}\left(s^{-1}\right)$ & $k_{2 c}\left(m^{3} \mathrm{~mol}^{-1} \mathrm{~s}^{-1}\right)$ & $\alpha_{1}(-)$ & $E_{\text {Ox/Re }}^{0}(V)$ \\
\hline 10.5 & 25.9 & 0.46 & 0.405 \\
\hline
\end{tabular}

Table 3

Parameters used in the simulations for the fits.

\begin{tabular}{ccccc}
\hline Electrodes & $3 \mathrm{HL}$ & $7 \mathrm{HL}$ & $11 \mathrm{HL}$ & $19 \mathrm{HL}$ \\
\hline$c_{E}^{t}\left(\mathrm{~mol} \mathrm{~m}^{-2}\right)$ & $8.9 \times 10^{-8}$ & $8.07 \times 10^{-8}$ & $5.7 \times 10^{-8}$ & $4.06 \times 10^{-8}$ \\
$L_{N}(\mu \mathrm{m})$ & 186 & 158 & 59 & 44 \\
\hline
\end{tabular}

\subsection{Voltammetry interpretation}

The templating procedure leads to a rather compact bead arrangement so that an FCC periodic cell represents a reasonable model for the resulting structure. This was observed in the work reported in (Reculusa and Ravaine, 2003) and further confirmed in a recent in-depth analysis of the 3D microstructure extracted from FIB-SEM imaging (Baux et al., 2020). The relative size of the pore connection window, $d_{c} / d_{s}$ was taken equal to $15 \%$, which can be considered as the minimum value for this manufacturing process (Szamocki et al., 2007). With these structural parameters, the effective diffusion coefficient was computed to be $\varepsilon_{f} D_{\text {eff }}^{*}=0.364$.

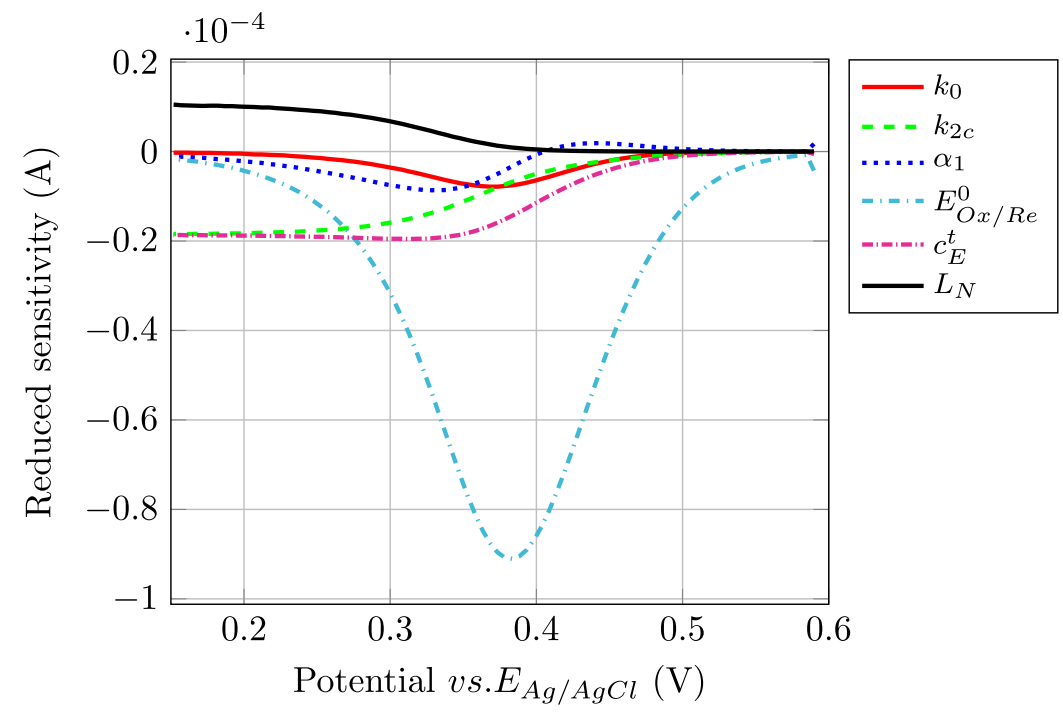

Fig. 9. Reduced sensitivities of the current to the parameters $k_{0}, k_{2 c}, \alpha_{1}, E_{O x / R e}^{0}, L_{N}$ and $c_{E}^{t}$ at their identified values on the $11 \mathrm{HL}$ electrode versus the scanning potential.

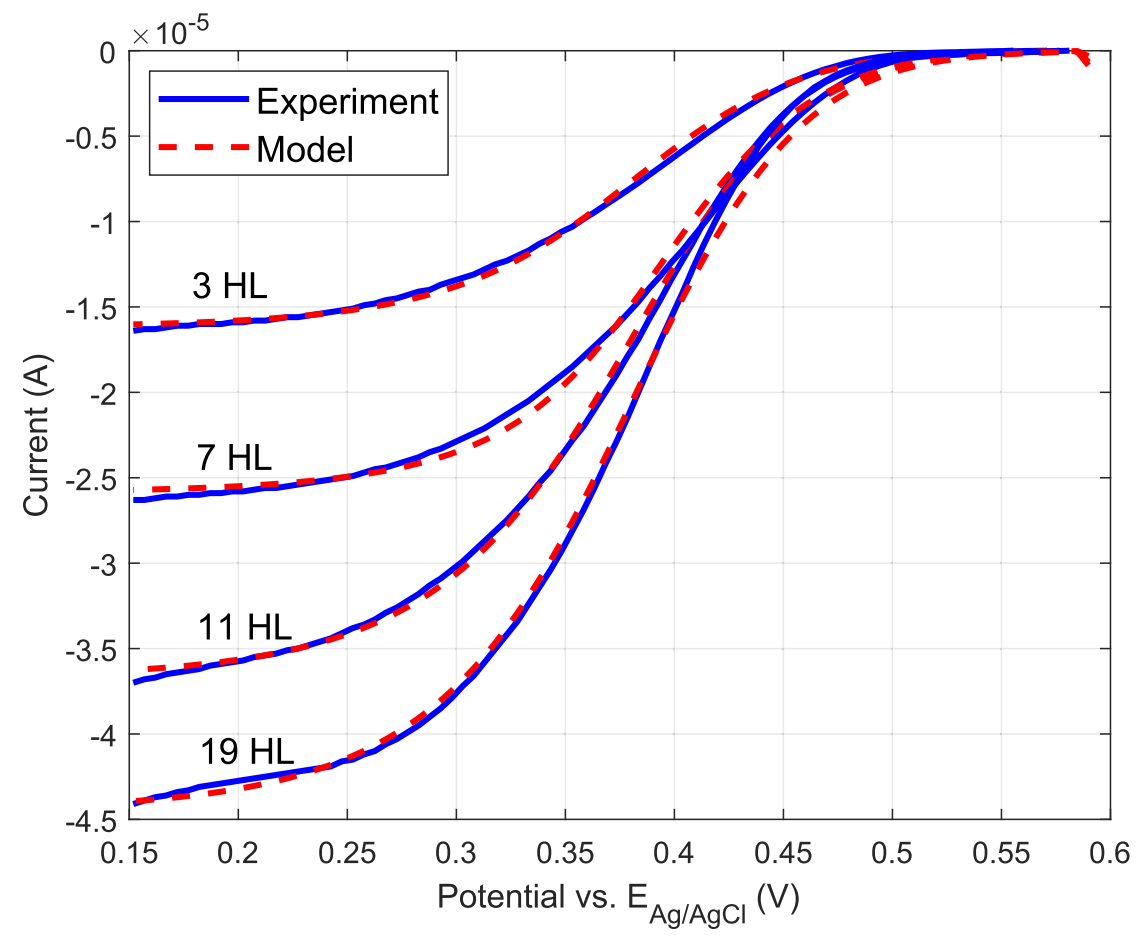

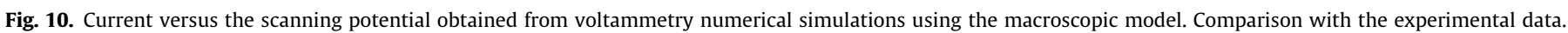




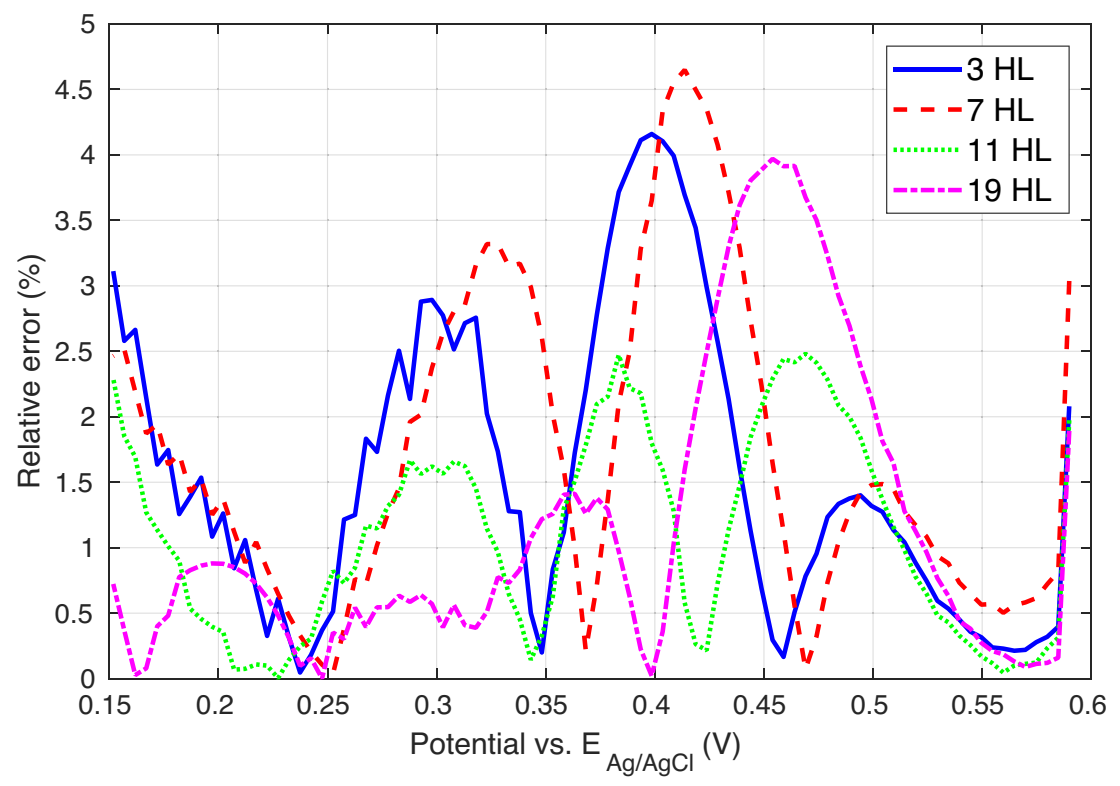

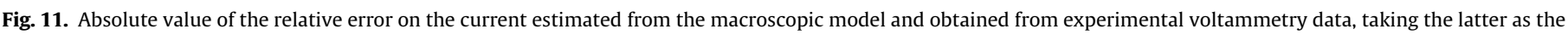
reference.

Interpretation of the experimental voltammetry data is carried out using a fitting procedure on some of the parameters involved in the macroscale model in Eqs. (17) that are unknown a priori. To do so, $k_{0}, k_{2 c}, \alpha_{1}$ and $E_{O x / r e}^{0}$ were estimated using a fitting procedure in the least square sense on data obtained with the $11 \mathrm{HL}$ electrode. It must be reminded that the individual values of $n_{2}$ and $\alpha_{2}$ are not required and that $k_{2 a}$ is taken equal to zero in agreement with the irreversibility of oxygen reduction ${ }^{1}$. The fitted resulting values are reported in Table 2 and were kept the same to predict the voltammetry results on the other electrodes (i.e., 3, 7 and 19HL).

Moreover, the total concentration of enzyme, $c_{E}^{t}$, and the diffusion layer thickness, $L_{N}$, that are subject to vary from one electrode to another, were fitted for each corresponding experimental data set. In fact, the diffusion layer thickness depends on the rate of oxygen depletion within the fluid in contact with the outer interface of the electrode, and this rate depends itself on the electrode thickness. Here, a simplified approach is adopted by considering $L_{N}$ as constant which means that this parameter should be understood as a mean value over the period of potential scanning for each experiment. The values are indicated in Table 3; those for the remaining parameters are given in Table 1 . It should be noted that the total enzyme surface concentration slightly decreases with the electrode thickness and this can be explained by the fact that, the thicker the porous material, the more difficult is the penetration of enzyme during the coating process deeper in the pores far from the free surface of the electrode. This observation was also part of the conclusions in the work by Do et al. (Do et al., 2014) on a somewhat different system. To assess the validity of the identification procedure, a sensitivity analysis of the current to all the fitted parameters was performed. The reduced sensitivity to a parameter, $u$, is defined as $u \partial I / \partial u$, $u$ being either $k_{0}, k_{2 c}, \alpha_{1}, E_{O x / R e}^{0}, L_{N}$ or $c_{E}^{t}$ here. It was computed using the $1 \mathrm{D}$ macroscopic model at the nominal values of each parameter identified on the 11 HL electrode. Sensitivities to all the parameters are represented versus the scanning potential in the interval of interest in Fig. 9. This figure clearly shows that all the sensitivities are significantly larger than the pre-

\footnotetext{
1 The value of $k_{2 a}$ was also identified in the fitting procedure and showed to be so small that it is insensitive in the current response.
}

cision of the potentiostat used to measure the current which can be estimated to be less than $0.0210^{-5} \mathrm{~A}$ over a very wide potential interval. In addition, the sensitivities are not proportional to each other, ensuring that parameters are uncorrelated. This analysis indicates that the identification procedure is robust and reliable.

The current versus the scanning potential obtained from our numerical simulation is represented in Fig. 10 together with the experimental results. As can be seen from this figure, excellent agreement is obtained for all the electrodes tested in this work over the whole scanned potential interval. In this potential interval, oxygen reduction on unmodified gold electrodes is not observed, because this reaction starts to occur only for potentials more negative than $0.1 \mathrm{~V}$ vs $\mathrm{Ag} / \mathrm{AgCl}$. Therefore, the signals should not be disturbed by such an eventual interference.

The experimental measurements show a slight crossover of the current signals at large potentials for the thickest electrodes $11 \mathrm{HL}$ and $19 \mathrm{HL}$ ). A possible explanation of this behavior might be that, at the very beginning of the bioelectrocatalytic reaction (onset potential of around $+0.5 \mathrm{~V}$ ), not all the enzyme modified layers are contributing immediately to the catalytic current for these two electrodes. Preferentially the outermost layers (most likely 2-3 half-layers, when looking at the relative positions of the plateau current for the 3 half-layer electrode $(15 \mu \mathrm{A})$ and the crossover current $(10 \mu A))$ are involved. Therefore the measured current is smaller with respect to what it should be in the ideal case when all the porous layers are active immediately. Only at higher driving forces $(E<+0.4 V)$, when the enzymes in the outer layers are already turning at their maximum speed, and thus cannot convert more oxygen per time unit, the inner layers start contributing as well because the oxygen diffusion layer now also reaches the inner part of the electrode. This leads to the observed crossover that is however not reproduced by the model which would require involving a concentrations-dependent catalytic rate constant. This effect is nevertheless of very weak importance.

The absolute value of the relative error between the numerical predictions and the experimental data, taking the latter as the reference, is less than 5\% as shown in Fig. 11 and is distributed around 0 . It remains even smaller than $3.5 \%$ when the potential is less than $\sim 0.37 V$ for all the electrodes. Moreover, the relative error when repeating several times the experiments is very small. 
For example, preparing three 7HL electrodes leads to electrocatalytic currents with a standard deviation of $0.0810^{-5} \mathrm{~A}$.

This successful comparison represents a strong validation step of the new macroscopic model derived in this work, assessing its relevance and performance to reproduce the physicoelectrochemical behavior of the porous electrodes operating in the DET mode.

\section{Conclusions}

In this work, a new multiscale modelling for diffusion and electrochemical enzymatic reaction involved in a porous electrode operating in the DET mode was developed. The unsteady porescale model was provided for oxygen reduction taking into account the electron transfer process at play. An upscaling procedure, in which coupling and non-linearities were handled, was applied to obtain a closed unsteady macroscopic model valid at the electrode scale. The associate closure problem, yielding the effective diffusivity tensor, was provided. This approach represents an original macroscopic model that has not been reported so far in the literature. In particular, unsteadiness, which has been disregarded in existing models for enzymatic porous electrodes, was explicitly taken into account. Moreover coupling between mass transport and enzymatic reaction in a non-linear way has been carried out through the upscaling procedure. This yields new terms induced by the enzymatic reaction in the macroscopic mass conservation equations (for enzyme and oxygen). Both the micro- and macroscale models were further used in the case of oxygen reduction in the presence of BOD as a catalyst. The successful comparison of numerical voltammetry simulations, carried out on a porous model structure with the 3D pore-scale model and the macroscale model which reduces to $1 \mathrm{D}$, assessed the validity of the latter. This macroscopic model was further employed to predict experimental voltammetry results obtained with porous gold electrodes coated with BOD. The excellent prediction confirms the relevance of the approach and the validity of this macroscopic model which provides an efficient tool operational at the electrode scale, allowing a considerable computational speedup. In particular, this model allows one to efficiently carry out a sensitivity analysis, which provides important indications on the most significant parameters involved in the physico-electrochemical process, a task that would be otherwise extremely cumbersome to perform with the porescale model. It also provides a mean to estimate parameters that are involved in the experiment (as for example the total enzyme concentration and standard rate constants). It could also be employed to interpret electrochemical impedance spectroscopy tests on electrodes for which dynamic effects are expected. More importantly, this model represents an essential tool for a rational optimal design of enzymatic porous electrodes. The multiscale approach developed here represents a breakthrough and could be widely used for other electrochemical processes in porous media.

\section{Declaration of Competing Interest}

The authors declare that they have no known competing financial interests or personal relationships that could have appeared to influence the work reported in this paper.

\section{Acknowledgement}

This work was supported by the LabEx AMADEus (ANR-10LABX-42) within IdEx Bordeaux (ANR-10-IDEX-03-02), i.e. the "Investissements d'Avenir Programme" of the French government managed by the Agence Nationale de la Recherche (ANR). It also benefited from the support of the ANR projects MOMA (ANR-17-
CE08-0005) and BIO3 (ANR- 16-CE19-0001). This project has also received partial funding from the European Union's Horizon 2020 research and innovation programme under the Marie Skłodowska-Curie grant agreement No 813006.

\section{Appendix A. Volume averaging and derivation of the upscaled model}

In this Appendix, the upscaling procedure to derive the macroscopic diffusion/reaction equation by volume averaging the microscopic initial boundary value problem (IBVP) given in Eqs. (5) is detailed. Volume averaging is applied according to the following four main steps (see Chap. 1 in (Whitaker, 1999 and ValdésParada et al., 2011)) and $c_{\mathrm{O}_{2}}$ is used to denote $c_{\mathrm{O}_{2}}(\mathbf{r}, t)$.

Step 1: Application of the superficial averaging operator

The superficial and area averages are defined as

$$
\begin{aligned}
& \left.\langle f\rangle\right|_{\mathbf{x}}=\left.\frac{1}{V} \int_{V_{f}(\mathbf{x})} f\right|_{\mathbf{x}+\mathbf{y}} \mathrm{d} V \\
& \left.\langle f\rangle_{s f}\right|_{\mathbf{x}}=\left.\frac{1}{A_{s f}} \int_{\mathrm{A}_{s f}(\mathbf{x})} f\right|_{\mathbf{x}+\mathbf{y}} \mathrm{d} A
\end{aligned}
$$

The superficial average operator is applied to the microscale IBVP and, with the purpose of deriving a model involving averages only, time and spatial derivation must be interchanged with volume averaging. This is achieved by using the general transport theorem, which, since the averaging volume is fixed in time and the porous medium is rigid, reduces in the present case to

$\left\langle\frac{\partial f}{\partial t}\right\rangle=\frac{\partial\langle f\rangle}{\partial t}$

and the spatial averaging theorem (or Leibnitz rule) given by (Truesdell and Toupin, 1960; Whitaker, 1999)

$\langle\nabla f\rangle=\nabla\langle f\rangle+\frac{1}{V} \int_{\mathrm{A}_{s f}} \mathbf{n} f d A$

together with a straightforward similar form for the divergence operator.

With this at hand, the superficial average can be applied to the mass balance Eq. (5d) for $\mathrm{O}_{2}$ and, employing the boundary condition at $\mathrm{A}_{s f}$, one arrives at the following average form

$$
\begin{array}{r}
\varepsilon_{f} \frac{\partial\left\langle c_{O_{2}}\right\rangle^{f}}{\partial t}=\nabla \cdot\left[\mathscr{D}_{O_{2}}\left(\varepsilon_{f} \boldsymbol{\nabla}\left\langle c_{O_{2}}\right\rangle^{f}+\left\langle c_{O_{2}}\right\rangle^{f} \nabla \varepsilon_{f}+\frac{1}{V} \int_{\mathrm{A}_{s f}} \mathbf{n} c_{O_{2}} \mathrm{~d} A\right)\right] \\
-k_{2 c} a_{v}\left\langle c_{O_{2}} c_{R e}\right\rangle_{s f}-k_{2 a} a_{v}\left\langle c_{R e}\right\rangle_{s f}+k_{2 a} a_{v} c_{E}^{t}
\end{array}
$$

where the intrinsic average concentration was used instead of the superficial average.

Step 2: Decomposition of $\mathrm{C}_{\mathrm{O}_{2}}$ and simplifications due to lengthscale constraints

The averaged Eq. (A.4) contains both average and point-wise concentrations. To remove the latter, the spatial decomposition

$c_{\mathrm{O}_{2}}=\left\langle c_{\mathrm{O}_{2}}\right\rangle^{f}+\tilde{c}_{\mathrm{O}_{2}}$

is introduced (Gray, 1975), where $\tilde{c}_{\mathrm{O}_{2}}$ is the deviation of concentration which fluctuates at a typical length-scale $\ell_{p}$ while $\left\langle c_{O_{2}}\right\rangle^{f}$ experiences significant variations at the scale $L$. It should be noted that a consequence of this decomposition is $\left\langle\tilde{c}_{\mathrm{O}_{2}}\right\rangle^{f}=0$, taking into account the scale hierarchy $\ell_{p} \ll L$. When this decomposition is inserted into Eq. (A.4), one gets

$$
\begin{aligned}
\varepsilon_{f} \frac{\partial\left\langle c_{O_{2}}\right\rangle^{f}}{\partial t}= & \nabla \cdot\left[\mathscr { D } _ { O _ { 2 } } \left(\varepsilon_{f} \boldsymbol{\nabla}\left\langle c_{O_{2}}\right\rangle^{f}+\left\langle c_{O_{2}}\right\rangle^{f} \nabla \varepsilon_{f}+\frac{1}{V} \int_{\mathrm{A}_{s f}} \mathbf{n}\left\langle c_{O_{2}}\right\rangle^{f} \mathrm{~d} A\right.\right. \\
& \left.\left.+\frac{1}{V} \int_{\mathrm{A}_{s f}} \mathbf{n} \tilde{c}_{O_{2}} \mathrm{~d} A\right)\right]-k_{2 c} a_{v}\left\langle c_{O_{2}} c_{R e}\right\rangle_{s f}-k_{2 a} a_{v}\left\langle c_{R e}\right\rangle_{s f}+k_{2 a} a_{v} c_{E}^{t}
\end{aligned}
$$


Attention must now be paid to the area integral term $\frac{1}{V} \int_{\mathrm{Asf}_{\mathrm{s}}} \mathbf{n}\left\langle c_{\mathrm{O}_{2}}\right\rangle^{f} \mathrm{~d} A$ on the right hand side of Eq. (A.6). This term must be evaluated at the centroid $\mathbf{x}$ of the averaging volume $\mathrm{V}$ and requires first the evaluation of $\left\langle c_{\mathrm{O}_{2}}\right\rangle^{f}$ at any point $\mathbf{x}+\mathbf{y}$ on $\mathrm{A}_{s f}$ contained in $\mathrm{V}$, making this term non-local. A Taylor expansion given by

$\left.\left\langle c_{O_{2}}\right\rangle^{f}\right|_{\mathbf{x}+\mathbf{y}}=\left.\left\langle c_{O_{2}}\right\rangle^{f}\right|_{\mathbf{x}}+\left.\mathbf{y} \cdot \nabla\left\langle c_{O_{2}}\right\rangle^{f}\right|_{\mathbf{x}}+\frac{1}{2} \mathbf{y y}:\left.\nabla \nabla\left\langle c_{O_{2}}\right\rangle^{f}\right|_{\mathbf{x}}+\ldots$

may be employed and, when introduced back into Eq. (A.6) together with the fact that

$$
\begin{aligned}
& \frac{1}{V} \int_{\mathrm{A}_{f s}} \mathbf{n} d A=-\nabla\langle 1\rangle=-\nabla \varepsilon_{f} \\
& \frac{1}{V} \int_{\mathrm{A}_{f s}} \mathbf{n y} d A=-\nabla\langle\mathbf{y}\rangle \\
& \frac{1}{V} \int_{\mathrm{A}_{f s}} \mathbf{n y y y} d A=-\nabla\langle\mathbf{y y}\rangle
\end{aligned}
$$

the average form of the mass conservation equation of species $A$ takes the form

$$
\begin{array}{r}
\varepsilon_{f} \frac{\partial\left\langle c_{O_{2}}\right\rangle^{f}}{\partial t}=\nabla \cdot\left[\mathscr { D } _ { O _ { 2 } } \left(\varepsilon_{f} \boldsymbol{\nabla}\left\langle c_{O_{2}}\right\rangle^{f}-\nabla\langle\mathbf{y}\rangle \cdot \nabla\left\langle c_{O_{2}}\right\rangle^{f}\right.\right. \\
\left.\left.-\frac{1}{2} \nabla\langle\mathbf{y y}\rangle: \nabla \nabla\left\langle c_{O_{2}}\right\rangle^{f}-\ldots+\frac{1}{V} \int_{\mathrm{A}_{s f}} \mathbf{n} \tilde{c}_{O_{2}} \mathrm{~d} A\right)\right] \\
-k_{2 c} a_{v}\left\langle c_{O_{2}} c_{R e}\right\rangle_{s f}-k_{2 a} a_{v}\left\langle c_{R e}\right\rangle_{s f}+k_{2 a} a_{v} c_{E}^{t}
\end{array}
$$

With the purpose of simplifying this last equation, orders of magnitude estimates can be employed to determine the important contributions among all the diffusive terms. Indeed, the following estimates can be derived

$$
\begin{aligned}
& \left.\varepsilon_{f} \nabla\left\langle c_{O_{2}}\right\rangle^{f}=\mathbf{0} \quad \varepsilon_{f} \frac{\left\langle c_{O_{2}}\right\rangle^{f}}{L}\right) \\
& \left.\nabla\langle\mathbf{y}\rangle \cdot \nabla\left\langle c_{O_{2}}\right\rangle^{f}=\mathbf{0} \quad \frac{\varepsilon_{f} r_{0}}{L} \frac{\left\langle c_{O_{2}}\right\rangle^{f}}{L}\right) \\
& \left.\nabla\langle\mathbf{y y}\rangle: \nabla \nabla\left\langle c_{O_{2}}\right\rangle^{f}=\mathbf{0}\left(\frac{\varepsilon_{f} r_{0}}{L}\right)^{2} \frac{\left\langle c_{O_{2}}\right\rangle^{f}}{L}\right)
\end{aligned}
$$

On the basis of the scale hierarchy, it is not hard to deduce that Eq. (A.11) can be simplified to

$$
\begin{array}{r}
\varepsilon_{f} \frac{\partial\left\langle c_{O_{2}}\right\rangle^{f}}{\partial t}=\boldsymbol{\nabla} \cdot\left[\mathscr{D}_{O_{2}}\left(\varepsilon_{f} \nabla\left\langle c_{O_{2}}\right\rangle^{f}+\frac{1}{V} \int_{\mathrm{A}_{s f}} \mathbf{n} \tilde{c}_{O_{2}} \mathrm{~d} A\right)\right] \\
-k_{2 c} a_{v}\left\langle c_{O_{2}} c_{R e}\right\rangle_{s f}-k_{2 a} a_{v}\left\langle c_{R e}\right\rangle_{s f}+k_{2 a} a_{v} c_{E}^{t}
\end{array}
$$

In order to progress to a form of this last expression involving $\left\langle c_{\mathrm{O}_{2}}\right\rangle^{f}$ only, the order of magnitude of $\tilde{c}_{\mathrm{O}_{2}}$ shall be obtained in comparison to that of $\left\langle c_{\mathrm{O}_{2}}\right\rangle^{f}$. To do so, B.C. 1 in Eq; (5e) can be rewritten as

$$
\begin{aligned}
-\mathbf{n} \cdot \mathscr{D}_{\mathrm{O}_{2}} \nabla \tilde{c}_{\mathrm{O}_{2}}-k_{2 c} \tilde{c}_{\mathrm{O}_{2}} c_{R e}= & \mathbf{n} \cdot \mathscr{D}_{\mathrm{O}_{2}} \nabla\left\langle c_{\mathrm{O}_{2}}\right\rangle^{f}+k_{2 c}\left\langle c_{\mathrm{O}_{2}}\right\rangle^{f} c_{R e} \\
& +k_{2 a} c_{R e}-k_{2 a} c_{E}^{t}
\end{aligned}
$$

Orders of magnitude of the deviation and average normal fluxes at $\mathrm{A}_{s f}$ can be estimated as

$$
\begin{aligned}
& \mathbf{n} \cdot \mathscr{D}_{\mathrm{O}_{2}} \nabla \tilde{c}_{\mathrm{O}_{2}}=\mathbf{O}\left(\mathscr{D}_{\mathrm{O}_{2}} \tilde{c}_{\mathrm{O}_{2}} / \ell_{p}\right) \\
& \mathbf{n} \cdot \mathscr{D}_{\mathrm{O}_{2}} \nabla\left\langle c_{\mathrm{O}_{2}}\right\rangle^{f}=\mathbf{O}\left(\mathscr{D}_{\mathrm{O}_{2}}\left\langle c_{\mathrm{O}_{2}}\right\rangle^{f} / L\right)
\end{aligned}
$$

This can be used in Eq. (A.14) to obtain an order of magnitude estimate of $\tilde{c}_{\mathrm{O}_{2}}$ given by

$$
\begin{aligned}
\tilde{c}_{O_{2}}= & \mathbf{0} \frac{\left(\ell_{p} / L\right)\left\langle c_{O_{2}}\right\rangle^{f}}{1+\mathbf{O}\left(k_{2 c} \ell_{p} c_{R e} / \mathscr{D}_{O_{2}}\right)}, \frac{\left(k_{2 c} \ell_{p} c_{R e} / \mathscr{D}_{\mathrm{O}_{2}}\right)\left\langle c_{\mathrm{O}_{2}}\right\rangle^{f}}{1+\mathbf{O}\left(k_{2 c} \ell_{p} c_{R e} / \mathscr{D}_{O_{2}}\right)}, \\
& \left.\times \frac{\left(k_{2 a} \ell_{p} c_{E}^{t} / \mathscr{D}_{\mathrm{O}_{2}}\right)}{1+\mathbf{O}\left(k_{2 c} \ell_{p} c_{R e} / \mathscr{D}_{O_{2}}\right)}\right)
\end{aligned}
$$

Noticing that $k_{2 a} \ll k_{2 c}$ and from the above estimate, it can be clearly seen that if the kinetic number, $K i$ is such that

$K i=\frac{k_{2 c} \ell_{p} c_{E}^{t}}{\mathscr{D}_{\mathrm{O}_{2}}} \ll 1$

then $\tilde{c}_{\mathrm{O}_{2}} \ll\left\langle c_{\mathrm{O}_{2}}\right\rangle^{f}$ at $\mathrm{A}_{\mathrm{sf}}$. Under these circumstances, Eq. (A.13) can be simplified to the following form

$$
\begin{aligned}
\varepsilon_{f} \frac{\partial\left\langle c_{O_{2}}\right\rangle^{f}}{\partial t}= & \nabla \cdot\left[\mathscr{D}_{O_{2}}\left(\varepsilon_{f} \nabla\left\langle c_{O_{2}}\right\rangle^{f}+\frac{1}{V} \int_{\mathrm{A}_{s f}} \mathbf{n} \tilde{c}_{O_{2}} \mathrm{~d} A\right)\right] \\
& -k_{2 c} a_{v}\left\langle\left\langle c_{O_{2}}\right\rangle^{f} c_{R e}\right\rangle_{s f}-k_{2 a} a_{v}\left\langle c_{R e}\right\rangle_{s f}+k_{2 a} a_{v} c_{E}^{t}
\end{aligned}
$$

In this equation, a close attention needs to be dedicated to the first of the three reactions terms which involves a non-local area integral. When the Taylor expansion of Eq. (A.7) is introduced in this integral, it comes

$\left\langle\left\langle c_{O_{2}}\right\rangle^{f} c_{R e}\right\rangle_{s f}=\left.\left\langle c_{R e}\right\rangle_{s f}\left\langle c_{O_{2}}\right\rangle^{f}\right|_{\mathbf{x}}+\left.\left\langle\mathbf{y} c_{R e}\right\rangle_{s f} \cdot \nabla\left\langle c_{O_{2}}\right\rangle^{f}\right|_{\mathbf{x}}+\frac{1}{2}\left\langle\mathbf{y} \mathbf{y} c_{R e}\right\rangle_{s f}:\left.\nabla \nabla\left\langle c_{O_{2}}\right\rangle^{f}\right|_{\mathbf{x}}+\ldots$

For the second and third terms on the right hand side, the following estimates can be made

$$
\begin{array}{r}
\left\langle\mathbf{y} c_{R e}\right\rangle_{s f} \ll r_{0}\left\langle c_{R e}\right\rangle_{s f} \\
\left\langle\mathbf{y y} c_{R e}\right\rangle_{s f}=r_{0}^{2}\left\langle c_{R e}\right\rangle_{s f}
\end{array}
$$

so that

$$
\begin{aligned}
& \left\langle\mathbf{y} c_{R e}\right\rangle_{s f} \cdot \nabla\left\langle c_{O_{2}}\right\rangle^{f} \ll \mathbf{O}\left(\frac{r_{0}}{L}\left\langle c_{O_{2}}\right\rangle^{f}\left\langle c_{R e}\right\rangle_{s f}\right) \ll\left\langle c_{O_{2}}\right\rangle^{f}\left\langle c_{R e}\right\rangle_{s f} \\
& \left\langle\mathbf{y y} c_{R e}\right\rangle_{s f}: \nabla \nabla\left\langle c_{O_{2}}\right\rangle^{f}=\mathbf{O}\left(\left(\frac{r_{0}}{L}\right)^{2}\right)\left\langle c_{O_{2}}\right\rangle^{f}\left\langle c_{R e}\right\rangle_{s f} \ll\left\langle c_{O_{2}}\right\rangle^{f}\left\langle c_{R e}\right\rangle_{s f}(A .2
\end{aligned}
$$

This shows that the dominant term in the expansion in Eq. (A.20) is $\left\langle c_{\mathrm{O}_{2}}\right\rangle^{f}\left\langle c_{\mathrm{Re}}\right\rangle_{s f}$. As a consequence the average Eq. (A.19) can be simplified to the following form

$$
\begin{aligned}
\varepsilon_{f} \frac{\partial\left\langle c_{O_{2}}\right\rangle^{f}}{\partial t}= & \nabla \cdot\left[\mathscr{D}_{O_{2}}\left(\varepsilon_{f} \nabla\left\langle c_{O_{2}}\right\rangle^{f}+\frac{1}{V} \int_{\mathrm{A}_{s f}} \mathbf{n} \tilde{c}_{O_{2}} \mathrm{~d} A\right)\right] \\
& -k_{2 c} a_{v}\left\langle c_{O_{2}}\right\rangle^{f}\left\langle c_{R e}\right\rangle_{s f}-k_{2 a} a_{v}\left\langle c_{R e}\right\rangle_{s f}+k_{2 a} a_{v} c_{E}^{t}
\end{aligned}
$$

The boundary condition at the solid-fluid interface (Eq. (A.14)) can be written as

$-\mathbf{n} \cdot \mathscr{D}_{\mathrm{O}_{2}} \nabla \tilde{c}_{\mathrm{O}_{2}}=\mathbf{n} \cdot \mathscr{D}_{\mathrm{O}_{2}} \nabla\left\langle c_{\mathrm{O}_{2}}\right\rangle^{f}+k_{2 c}\left\langle c_{O_{2}}\right\rangle^{f} c_{R e}+k_{2 a} c_{R e}-k_{2 a} c_{E}^{t}$

In addition, taking into account the above order of magnitude estimate, the averaged mass conservation equation for the enzyme is given by

$$
\frac{\partial\left\langle c_{R e}\right\rangle_{s f}}{\partial t}=-\left(k_{1 c}+k_{1 a}+k_{2 a}\right)\left\langle c_{R e}\right\rangle_{s f}-k_{2 c}\left\langle c_{R e}\right\rangle_{s f}\left\langle c_{O_{2}}\right\rangle^{f}+\left(k_{1 c}+k_{2 a}\right) c_{E}^{t}
$$

At this stage, the model remains unclosed since $\tilde{c}_{\mathrm{O}_{2}}$ is still present in the averaged mass balance Eq. (A.23c).

\section{Step 3: Closure}

The idea is now to form an IBVP (i.e. a closure problem) on $\tilde{c}_{\mathrm{O}_{2}}$ which solution can be inserted into the averaged equation in order to get a closed macroscopic model. This can be achieved by subtracting Eq. (A.23c) divided by $\varepsilon_{f}$ and Eq. (A.23e) from their corresponding pore-scale equivalents in Eqs. (5d) and (5a), respectively and by using the boundary condition under the form of Eq. (A.23d). Moreover, since the purpose is not to solve the closure problem on the entire structure of characteristic length-scale $L$ but, rather, on a representative unit cell of the structure of characteristic lengthscale $\ell_{R}$, the external boundary condition shall be replaced by a 
periodic boundary condition on $\tilde{c}_{\mathrm{O}_{2}}$ (see Chap. 1 in (Whitaker, 1999)), yielding

$$
\begin{aligned}
\frac{\partial \tilde{c}_{R}}{\partial t}= & -\left(k_{1 c}+k_{1 a}+k_{2 a}+k_{2 c}\left\langle c_{O_{2}}\right\rangle^{f}\right) \tilde{c}_{R}-k_{2 c} \tilde{c}_{O_{2}}\left\langle c_{R e}\right\rangle_{s f} \\
\frac{\partial \tilde{c}_{O_{2}}}{\partial t}= & \nabla \cdot\left(\mathscr{D}_{O_{2}} \nabla \tilde{c}_{O_{2}}\right)-\varepsilon_{f}^{-1} \mathscr{D}_{O_{2}} \nabla \varepsilon_{f} \cdot \nabla\left\langle c_{O_{2}}\right\rangle^{f}-\varepsilon_{f}^{-1} \nabla \cdot\left(\frac{\mathscr{D}_{O_{2}}}{V} \int_{\mathrm{A}_{s f}} \mathbf{n} \tilde{c}_{O_{2}} \mathrm{~d} A\right) \\
& +\varepsilon_{f}^{-1} k_{2 c} a_{v}\left\langle c_{O_{2}}\right\rangle^{f}\left\langle c_{R e}\right\rangle_{s f}+\varepsilon_{f}^{-1} k_{2 a} a_{v}\left(\left\langle c_{R e}\right\rangle_{s f}-c_{E}^{t}\right) \text { in } \mathrm{V}_{f} \quad(\mathrm{~A} .27 \mathrm{~b}) \\
-\mathbf{n} \cdot \mathscr{D}_{O_{2}} \nabla \tilde{c}_{O_{2}}=\mathbf{n} \cdot \mathscr{D}_{O_{2}} \nabla\left\langle c_{O_{2}}\right\rangle^{f}+k_{2 c}\left\langle c_{O_{2}}\right\rangle^{f} c_{R e}+k_{2 a} c_{R e}-k_{2 a} c_{E}^{t} & \text { at } \mathrm{A}_{s f} \\
\tilde{c}_{O_{2}}(\mathbf{r}, t)= & \tilde{c}_{O_{2}}\left(\mathbf{r}+\ell_{R} \mathbf{e}_{i}, t\right)
\end{aligned}
$$

to which an initial condition must be added. Here $\mathbf{e}_{i}$ denotes one of the unit basis vectors. To obtain Eq. (A.27a), the decomposition $c_{R e}=\left\langle c_{R e}\right\rangle_{s f}+\tilde{c}_{R}$ was employed in conjunction with the fact that $\tilde{c}_{\mathrm{O}_{2}} \ll\left\langle c_{\mathrm{O}_{2}}\right\rangle^{f}$.

To simplify this problem, the following order of magnitude estimates can be employed

$\left.\boldsymbol{\nabla} \cdot\left(\mathscr{D}_{\mathrm{O}_{2}} \nabla \tilde{C}_{\mathrm{O}_{2}}\right)=\mathbf{0} \frac{\mathscr{D}_{\mathrm{O}_{2}} \tilde{\mathrm{C}}_{\mathrm{O}_{2}}}{\ell_{p}^{2}}\right)$

$\left.\varepsilon_{f}^{-1} \nabla \cdot \frac{\mathscr{D}_{\mathrm{O}_{2}}}{V} \int_{\mathrm{A}_{s f}} \mathbf{n} \tilde{\boldsymbol{c}}_{\mathrm{O}_{2}} \mathrm{~d} A\right)=\mathbf{0}\left(\frac{\mathscr{D}_{\mathrm{O}_{2}} \tilde{\mathrm{C}}_{\mathrm{O}_{2}}}{\varepsilon_{f} L \ell_{p}}\right)$

which, based on the length-scale hierarchy, indicates that the latter term is negligible with respect to the former. Moreover, the diffusive volume source $\varepsilon_{f}^{-1} \mathscr{D}_{\mathrm{O}_{2}} \nabla \varepsilon_{f} \cdot \nabla\left\langle c_{\mathrm{O}_{2}}\right\rangle^{f}$ and of the diffusive surface source $\mathbf{n} \cdot \mathscr{D}_{\mathrm{O}_{2}} \nabla\left\langle c_{\mathrm{O}_{2}}\right\rangle^{f}$ can be compared by analyzing their respective contribution per unit volume of the porous medium. The corresponding order of magnitude estimates are given by

$\frac{1}{V} \int_{\mathrm{V}_{f}} \varepsilon_{f}^{-1} \mathscr{D}_{\mathrm{O}_{2}} \nabla \varepsilon_{f} \cdot \nabla\left\langle c_{O_{2}}\right\rangle^{f} \mathrm{~d} V=\mathbf{0}\left(\frac{\varepsilon_{f} \mathscr{D}_{O_{2}}}{L^{2}}\left\langle c_{O_{2}}\right\rangle^{f}\right)$
$\bar{V} \int_{\mathrm{A}_{s f}} \mathbf{n} \cdot \mathscr{D}_{\mathrm{O}_{2}} \nabla\left\langle c_{\mathrm{O}_{2}}\right\rangle^{f} \mathrm{~d} A=\mathbf{0}\left(\frac{\mathscr{D}_{\mathrm{O}_{2}}}{\ell_{p} L}\left\langle c_{\mathrm{O}_{2}}\right\rangle^{f}\right)$

showing that the diffusive volume source has a negligible contribution. In addition, when the process is considered at a time scale constrained by

$\left.t \gg \max \frac{\ell_{p}^{2}}{\mathscr{D}_{\mathrm{O}_{2}}}, \frac{1}{k_{1 c}+k_{1 a}+k_{2 a}+k_{2 c}\left\langle c_{O_{2}}\right\rangle^{f}}\right)$

it is not hard to deduce that both unsteady terms can be neglected in Eqs. (A.27a) and (A.27b) so that the closure problem becomes steady. Finally, since $\tilde{c}_{O_{2}} \ll\left\langle c_{O_{2}}\right\rangle^{f}$, it follows that $k_{2 c} \tilde{c}_{O_{2}} \ll k_{1 c}+k_{1 a}+k_{2 a}+k_{2 c}\left\langle c_{O_{2}}\right\rangle^{f}$. As a consequence, Eq. (A.27a) yields $\tilde{c}_{R e} \ll\left\langle c_{R e}\right\rangle_{s f}$ and the closure problem takes the form

$$
\begin{aligned}
& \nabla^{2} \tilde{c}_{O_{2}}=-\frac{\varepsilon_{f}^{-1} k_{2 c} a_{v}}{\mathscr{D}_{O_{2}}}\left\langle c_{O_{2}}\right\rangle^{f}\left\langle c_{R e}\right\rangle_{s f}-\frac{\varepsilon_{f}^{-1} k_{2 a} a_{v}}{\mathscr{D}_{O_{2}}}\left(\left\langle c_{R e}\right\rangle_{s f}-c_{E}^{t}\right) \\
& -\mathbf{n} \cdot \mathscr{D}_{\mathrm{O}_{2}} \nabla \tilde{c}_{\mathrm{O}_{2}}=\mathbf{n} \cdot \mathscr{D}_{\mathrm{O}_{2}} \nabla\left\langle c_{\mathrm{O}_{2}}\right\rangle^{f}+k_{2 c}\left\langle c_{\mathrm{O}_{2}}\right\rangle^{f}\left\langle c_{\mathrm{Re}}\right\rangle_{s f} \\
& +k_{2 a}\left(\left\langle c_{R e}\right\rangle_{s f}-c_{E}^{t}\right) \text { at } \mathrm{A}_{s f} \\
& \tilde{c}_{\mathrm{O}_{2}}(\mathbf{r})=\tilde{c}_{\mathrm{O}_{2}}\left(\mathbf{r}+\ell_{\mathrm{rev}} \mathbf{e}_{i}\right)
\end{aligned}
$$

This problem has a linear structure, so the solution on $\tilde{c}_{\mathrm{O}_{2}}$ can be sought in terms of a linear combination of the sources under the form

$\tilde{c}_{O_{2}}=\mathbf{b} \cdot \nabla\left\langle c_{O_{2}}\right\rangle^{f}+s\left\langle c_{O_{2}}\right\rangle^{f}\left\langle c_{R e}\right\rangle_{s f}+h\left(\left\langle c_{R e}\right\rangle_{s f}-c_{E}^{t}\right)$ b, $s$ and $h$ being the closure variables (note that $\mathbf{b}$ is a vector whereas $s$ and $h$ are scalars) which can be chosen to obey the following boundary value problems

Problem I

$\nabla^{2} \mathbf{b}=0 \quad$ in $\mathrm{V}_{f}$

$\mathbf{n} \cdot \nabla \mathbf{b}=-\mathbf{n} \quad$ at $A_{s f}$

$\langle\mathbf{b}\rangle^{f}=0$

$\mathbf{b}(\mathbf{r})=\mathbf{b}\left(\mathbf{r}+\ell_{\text {rev }} \mathbf{e}_{i}\right)$

Problem II

$\nabla^{2} s=-\frac{\varepsilon_{f}^{-1} k_{2 c} a_{v}}{\mathscr{D}_{0_{2}}} \quad$ in $\mathrm{V}_{f}$

n. $\nabla s=-\frac{k_{2 c}}{\mathscr{D}_{\mathrm{O}_{2}}} \quad$ at $\mathrm{A}_{s f}$

$\langle s\rangle^{f}=0$

$s(\mathbf{r})=s\left(\mathbf{r}+\ell_{r e v} \mathbf{e}_{i}\right)$

Problem III

$\nabla^{2} h=-\frac{\varepsilon_{f}^{-1} k_{2 a} a_{v}}{\mathscr{D}_{O_{2}}} \quad$ in $\mathrm{V}_{f}$

$\mathbf{n} \cdot \nabla h=-\frac{k_{2 a}}{\mathscr{D}_{\mathrm{O}_{2}}} \quad$ at $\mathrm{A}_{s f}$

$\langle h\rangle^{f}=0$

$h(\mathbf{r})=h\left(\mathbf{r}+\ell_{\text {rev }} \mathbf{e}_{i}\right)$

Note that the conditions in Eqs. (A.33c), (A.34c) and (A.35c) result from the fact that $\left\langle\tilde{C}_{\mathrm{O}_{2}}\right\rangle^{f}=0$ and are required for both closure problems to have a unique solution.

Step 4: Macroscopic model

When the representation of $\tilde{C}_{\mathrm{O}_{2}}$ is reported back into the average Eq. (A.23c), one finally obtains the following closed macroscopic equation for $\left\langle c_{\mathrm{O}_{2}}\right\rangle^{f}$

$$
\begin{aligned}
\varepsilon_{f} \frac{\partial\left\langle c_{O_{2}}\right\rangle^{f}}{\partial t} & =\boldsymbol{\nabla} \cdot\left(\varepsilon_{f} \mathbf{D}_{e f f} \cdot \nabla\left\langle c_{O_{2}}\right\rangle^{f}\right)+\nabla \cdot\left(\mathbf{s}\left\langle c_{O_{2}}\right\rangle^{f}\left\langle c_{R e}\right\rangle_{s f}\right) \\
& +\nabla \cdot\left(\mathbf{h}\left(\left\langle c_{R e}\right\rangle_{s f}-c_{E}^{t}\right)\right) \\
& -k_{2 c} a_{v}\left\langle c_{O_{2}}\right\rangle^{f}\left\langle c_{R e}\right\rangle_{s f}-k_{2 a} a_{v}\left\langle c_{R e}\right\rangle_{s f}+k_{2 a} a_{v} c_{E}^{t}
\end{aligned}
$$

with the effective parameters $\mathbf{D}_{\text {eff }}$ and $\mathbf{s}$ and $\mathbf{h}$ given by

$$
\begin{aligned}
& \left.\mathbf{D}_{\text {eff }}=\mathscr{D}_{\mathrm{O}_{2}} \mathbf{I}+\frac{1}{V_{f}} \int_{\mathrm{A}_{s f}} \mathbf{n b} d A\right) \\
& \mathbf{s}=\frac{\mathscr{D}_{\mathrm{O}_{2}}}{V}\left(\int_{\mathrm{A}_{\mathrm{sf}}} \mathbf{n} s d A\right) \\
& \mathbf{h}=\frac{\mathscr{D}_{\mathrm{O}_{2}}}{V}\left(\int_{\mathrm{A}_{s f}} \mathbf{n} h d A\right)
\end{aligned}
$$

where $\mathbf{b}, s$ and $h$ are solution of the closure problems $I, I I$ and $I I I$ in Eqs. (A.33), (A.34) and (A.35) respectively. Note that in Eq. (A.37), $\mathbf{D}_{\text {eff }}$ is a second order tensor and $\mathbf{I}$ is the identity; $\mathbf{n b}$ denotes the outer product between the two vectors $\mathbf{n}$ and $\mathbf{b}$.

From Eq. (A.34b), the order of magnitude of $s$ can be estimated to be $s=\mathbf{O}\left(k_{2 c} \ell_{p} / \mathscr{D}_{\mathrm{O}_{2}}\right)$ and from Eq. (A.38), one gets $\mathbf{s}=\mathbf{O}\left(k_{2 c}\right)$. This leads to an order of magnitude estimate for the second term on the right hand side of Eq. (A.36) given by $\boldsymbol{\nabla} \cdot\left(\mathbf{s}\left\langle c_{\mathrm{O}_{2}}\right\rangle^{f}\left\langle c_{\mathrm{Re}}\right\rangle_{s f}\right)=$ $\mathbf{0}\left(\frac{k_{2 c}}{L}\left\langle c_{\mathrm{O}_{2}}\right\rangle^{f}\left\langle c_{\mathrm{Re}}\right\rangle_{s f}\right)$ while the order of magnitude of the macroscopic reactive term is $k_{2 c} a_{v}\left\langle c_{O_{2}}\right\rangle^{f}\left\langle c_{R e}\right\rangle_{s f}=\mathbf{O}\left(\frac{k_{2 c}}{\ell_{p}}\left\langle c_{O_{2}}\right\rangle^{f}\left\langle c_{R e}\right\rangle_{s f}\right)$ with the idea that $a_{v}=\mathbf{O}\left(\ell_{p}^{-1}\right)$. The same estimate can be made for $\mathbf{h}$. On the 
basis of the scale hierarchy, this indicates that the macroscopic diffusion/reaction equation for $\left\langle c_{0_{2}}\right\rangle^{f}$ may finally be written as

$$
\begin{aligned}
\varepsilon_{f} \frac{\partial\left\langle c_{O_{2}}\right\rangle^{f}}{\partial t}= & \nabla \cdot\left(\varepsilon_{f} \mathbf{D}_{e f f} \cdot \nabla\left\langle c_{O_{2}}\right\rangle^{f}\right)-k_{2 c} a_{v}\left\langle c_{O_{2}}\right\rangle^{f}\left\langle c_{R e}\right\rangle_{s f} \\
& -k_{2 a} a_{v}\left\langle c_{R e}\right\rangle_{s f}+k_{2 a} a_{v} c_{E}^{t}
\end{aligned}
$$

The average equation for $\left\langle c_{R e}\right\rangle_{s f}$ is given by Eq. (A.23e).

\section{References}

Al-Lolage, F., Bartlett, P., Gounel, S., Staigre, P., Mano, N., 2019. Site-directed immobilization of bilirubin oxidase for electrocatalytic oxygen reduction. ACS Catal. 9 (3), 2068-2078.

Ban, Z., Katelhon, E., Compton, R.G., 2016. Voltammetry of porous layers: Staircase vs analog voltammetry. J. Electroanal. Chem. 776, 25-33.

Barcia, O.E., D’Elia, E., Frateur, I., Mattos, O.R., Pebere, N., Tribollet, B., 2002. Application of the impedance model of de Levie for the characterization of porous electrodes. Electrochim. Acta 47, 2109-2116.

Barnes, E.O., Chen, X., Li, P., Compton, R.G., 2014. Voltammetry at porous electrodes: A theoretical study. J. Electroanal. Chem. 720-721, 92-100.

Bartlett, P., Pratt, K., 1995. Theoretical treatment of diffusion and kinetics in amperometric immobilized enzyme electrodes part i: Redox mediator entrapped within the film. J. Electroanal. Chem. 397, 61-78.

Bartlett, P.N., Birkina, P.R., Ghanema, M.A., 2000. Electrochemical deposition of macroporous platinum, palladium and cobalt films using polystyrene latex sphere templates. Chem. Commun. 17, 1671-1672.

Baux, A., Couégnat, G., Vignoles, G., Lasseux, D., Kuhn, A., Carucci, C., Mano, N., Le, T., 2020. Digitization and image-based structure-properties relationship evaluation of a porous gold micro-electrode. Mater. Des. 193, 108812.

Blodgett, K.B., 1935. Films built by depositing successive monomolecular layers on a solid surface. J. Am. Chem. Soc. 57, 1007-1022.

Brocato, S., Lau, C., Atanassov, P., 2012. Mechanistic study of direct electron transfer in bilirubin oxidase. Electrochim. Acta 61, 44-49.

Butler, J.A.V., 1932. The mechanism of overvoltage and its relation to the combination of hydrogen atoms at metal electrodes. Trans. Faraday Soc. 28, 379-382.

Cadet, M., Gounel, S., Stines-Chaumeil, C., Brilland, X., Rouhana, J., Louerat, F., Mano, N., 2016. An enzymatic glucose $/ \mathrm{O}_{2}$ biofuel cell operating in human blood. Biosens. Bioelectron. 83, 60-67.

Chan, H.T.H., Katelhon, E., Compton, R.G., 2017. Voltammetry using multiple cycles: Porous electrodes. J. Electroanal. Chem. 799, 126-133.

Cosnier, S., Gross, A.J., Goff, A.L., Holzinger, M., 2016. Recent advances on enzymatic glucose/oxygen and hydrogen/oxygen biofuel cells: Achievements and limitations. J. Power Sources 325, 252-263.

de Poulpiquet, A., Ranava, D., Monsalve, K., Giudici-Orticoni, M.T., Lojou, E., 2014. Biohydrogen for a new generation of $\mathrm{H}_{2} / \mathrm{O}_{2}$ biofuel cells: A sustainable energy perspective. ChemElectroChem 1, 1724-1750.

de Poulpiquet, A., Ciaccafava, A., Lojou, E., 2014. New trends in enzyme immobilization at nanostructured interfaces for efficient electrocatalysis in bio fuel cells. Electrochim. Acta 126, 104-114.

Do, T.Q.N., Varničić, M., Hanke-Rauschenbach, R., Vidaković-Koch, T., Sundmacher, K., 2014. Mathematical modeling of a porous enzymatic electrode with direct electron transfer mechanism. Electrochim. Acta 137, 616-626.

Ender, M., 2015. An extended homogenized porous electrode model for lithium-ion cell electrodes. J. Power Sources 282, 572-580.

Ferguson, T.R., Bazant, M.Z., 2012. Nonequilibrium thermodynamics of porous electrodes. J. Electrochem. Soc. 159, 1967-1985.

Galceran, J., Taylor, S., Bartlett, P., 2001. Modelling the steady-state current at the inlaid disc microelectrode for homogeneous mediated enzyme catalysed reactions. J. Electroanal. Chem. 506, 65-81.

Gamella, M., Koushanpour, A., Katz, E., 2018. Biofuel cells - activation of micro- and macro-electronic devices. Bioelectrochemistry 119, 33-42.

Gray, W.G., 1975. A derivation of the equations for multi-phase transport. Chem. Eng. Sci. 30, 229-233.

Hexter, S.V., Grey, F., Happe, T., Climent, V., Armstrong, F.A., 2012. Electrocatalytic mechanism of reversible hydrogen cycling by enzymes and distinctions between the major classes of hydrogenases. PNAS 109, 11516-11521.

Hexter, S.V., Esterle, T.F., Armstrong, F.A., 2014. A unified model for surface electrocatalysis based on observations with enzymes. PCCP 16, 11822-11833.

Karajić, A., Reculusa, S., Ravaine, S., Mano, N., Kuhn, A., 2016. Miniaturized electrochemical device from assembled cylindrical macroporous gold electrodes. ChemElectroChem 03, 2031-2035.

Lasseux, D., Valdés-Parada, F.J., 2017. Symmetry properties of macroscopic transport coefficients in porous media. Phys. Fluids 29 (4), 043303.

Le, T., Lasseux, D., 2020. Current and optimal thickness predictions for a porous micro-electrode. ChemElectroChem 7, 3017-3027.

Leech, D., Kavanagh, P., Schuhmann, W., 2012. Enzymatic fuel cells: Recent progress. Electrochim. Acta 84, 223-234.
Le, T.D., Lasseux, D., Nguyen, X.P., Vignoles, G.L., Mano, N., Kuhn, A., 2017. Multiscale modeling of diffusion and electrochemical reactions in porous microelectrodes. Chem. Eng. Sci. 173, 153-167.

Le, T.D., Zhang, L., Vignoles, G.L., Mano, N., Kuhn, A., Lasseux, D., 2019. Optimal thickness of a porous micro-electrode operating a single redox reaction. ChemElectroChem 6, 173-180.

Le, T.D., Zhang, L., Kuhn, A., Mano, N., Vignoles, G.L., Lasseux, D., 2019. Upscaled model for diffusion and serial reduction pathways in porous electrodes. J. Electroanal. Chem. 855, 113325.

Levie, R.D., 1967. Electrochemical response of porous and rough electrodes, Advances in Electrochemistry and Electrochemical. Engineering 6, 329-397.

Mano, N., de Poulpiquet, A., 2018. $\mathrm{o}_{2}$ reduction in enzymatic biofuel cells. Chem. Rev. 118 (5), 2392-2468.

Mazurenko, I., Monsalve, K., Rouhana, J., Parent, P., Laffon, C., Goff, A.L., Szunerits, S., Boukherroub, R., Giudici-Orticoni, M.T., Mano, N., Lojou, E., 2016. How the intricate interactions between carbon nanotubes and two bilirubin oxidases control direct and mediated O_2 reduction. Applied Materials and Interfaces 8, 23074-23085.

Minteer, S.D., Atanassov, P., Luckarift, H.R., Johnson, G.R., 2012. New materials for biological fuel cells. Materialstoday 15, 166-173.

Moehlenbrock, M.J., Minteer, S.D., 2008. Extended lifetime biofuel cells. Chem. Soc. Rev. 37, 1188-1196.

Rajendran, L., Kirthiga, M., Laborda, E., 2017. Mathematical modeling of nonlinear reaction-diffusion processes in enzymatic biofuel cells. Current Opinion in Electrochemistry 1 (1), 121-132.

Rasmussen, M., Abdellaoui, S., Minteer, S.D., 2016. Enzymatic biofuel cells: 30 years of critical advancements. Biosens. Bioelectron. 76, 91-102.

Reculusa, S., Heim, M., Garrigue, P., Ravaine, S., Mano, N., Kuhn, A.PLEASE CORRECT Karaji TO Karajic (WITH AN ACCENT ON THE c), 2015. Bottom-up generation of miniaturized coaxial double electrodes with tunable porosity. Adv. Mater. Interfaces 2 (12), 1500192-1500196.

Reculusa, S., Ravaine, S., 2003. Synthesis of colloidal crystals of controllable thickness through the Langmuir-Blodgett technique. Chem. Mater. 15, 598-605.

Reculusa, S., Heim, M., Gao, F., Mano, N., Ravaine, S., Kuhn, A., 2011. Design of catalytically active cylindrical and macroporous gold microelectrodes. Adv. Funct. Mater. 21 (4), 691-698.

Shleev, S., 2017. Quo vadis, implanted fuel cell? ChemPlusChem 82, 522-539.

Siepenkoetter, T., Salaj-Kosla, U., Xiao, X., Conghaile, P., Pita, M., Ludwig, R., Magner, E., 2017. Immobilization of redox enzymes on nanoporous gold electrodes: Applications in biofuel cells. ChemPlusChem 82 (4), 553-560.

Solomon, E., Sundaram, U., Machonkin, T., 1996. Multicopper oxidases and oxygenases. Chem. Rev. 96, 2563-2605.

Stines-Chaumeil, C., Roussarie, E., Mano, N., 2017. The nature of the rate-limiting step of blue multicopper oxidases: Homogeneous studies versus heterogeneous. Biochim. Open 4, 36-40.

Szamocki, R., Reculusa, S., Ravaine, S., Bartlett, P.N., Kuhn, A., Hempelmann, R., 2006. Tailored mesostructuring and biofunctionalization of gold for increased electroactivity. Angew. Chem. Int. Ed. 45 (8), 1317-1321.

Szamocki, R., Velichko, A., Mücklich, F., Reculusa, S., Ravaine, S., Neugebauer, S., Schuhmann, W., Hempelmann, R., Kuhn, A., 2007. Improved enzyme immobilization for enhanced bioelectrocatalytic activity of porous electrodes. Electrochem. Commun. 9, 2121-2127.

Truesdell, C., Toupin, R., 1960. The classical field theories. Springer-Verlag, NewYork.

Tsujimura, S., Nakagawa, T., Kano, K., Ikeda, T., 2004. Kinetic study of direct bioelectrocatalysis of dioxygen reduction with bilirubin oxidase at carbon electrodes. Electrochemistry 72, 437-439.

Valdés-Parada, F., Aguilar-Madera, C., Álvarez Ramírez, J., 2011. On diffusion, dispersion and reaction in porous media. Chem. Eng. Sci. 66, 2177-2190.

Valdés-Parada, F.J., Lasseux, D., Whitaker, S., 2017. Diffusion and heterogeneous reaction in porous media: The macroscale model revisited. Int. J. Chem. Reactor Eng. 152017 (6). https://doi.org/10.1515/ijcre-2017-0151.

Valdés-Parada, F.J., Lasseux, D., Whitaker, S., 2020. Upscaling reactive transport under hydrodynamic slip conditions in homogeneous porous media. Water Resour. Res. 56 (1). https://doi.org/10.1029/2019WR025954. e2019WR025954.

Vidts, P.D., White, R.E., 1997. Governing equations for transport in porous electrodes. J. Electrochem. Soc. 144, 1343-1352.

Walcarius, A., Kuhn, A., 2008. Ordered porous thin films in electrochemical analysis. Trends Anal. Chem. 27, 593-603.

Wang, L., Wu, X., Su, B.-W., Song, R., Zhang, J.-R., Zhu, J.-J., 2021. Enzymatic biofuel cell: Opportunities and intrinsic challenges in futuristic applications. Adv. Energy Sustainability Res., 2100031

Whitaker, S., 1999. The method of volume averaging. Kluwer Academic Publishers, Dordrecht, The Netherlands.

Xiao, X., Xia, H.-Q., Wu, R., Bai, L., Yan, L., Magner, E., Cosnier, S., Lojou, E., Zhu, Z., Liu, A., 2019. Tackling the challenges of enzymatic (bio)fuel cells. Chem. Rev. 119 (16), 9509-9558.

Zhang, L., Carucci, C., Reculusa, S., Goudeau, B., Lefrancois, P., Gounel, S., Mano, N., Kuhn, A., 2019. Rational design of enzyme modified electrodes for optimized bioelectrocatalytic activity. ChemElectroChem. in press.doi:10.1002/ celc. 201901022 .

Zhao, C.E., Gai, P., Song, R., Chen, Y., Zhang, J., Zhu, J.J., 2017. Nanostructured material-based biofuel cells: recent advances and future prospects. Chem. Soc. Rev. 46 (5), 1545-1564. 